\title{
Significant enhancements of secondary cosmic rays and electric field at the high mountain peak of Lomnický Štít in High Tatras during thunderstorms
}

\author{
J. Chum ${ }^{1 *} \mathbb{B}$, R. Langer ${ }^{2,4}$, J. Baše ${ }^{1}$, M. Kollárik², I. Strhárský², G. Diendorfer ${ }^{3}$ and J. Rusz ${ }^{1}$
}

\begin{abstract}
High electric fields that occur in thunderstorm clouds in the Earth's atmosphere might accelerate energetic charged particles produced by cosmic rays. Such energetic particles, especially electrons, can cause additional ionization as they are multiplied and thus form avalanche of relativistic electrons. These relativistic electrons emit Bremsstrahlung in the X- or gamma-ray spectral ranges as they lose their kinetic energy via collisions. Thunderstorm ground enhancements (TGEs) of secondary cosmic ray fluxes recorded at the top of a sharp rocky mountain of Lomnický Štít in High Tatras (2634 m, Slovak Republic) are compared with simultaneous measurements of electric field at the mountain top and on its slope at the observatory of Skalnaté Pleso $(1780 \mathrm{~m})$. Results of measurements performed from May to September in 2017 and from May to October in 2018 are presented. The cosmic ray flux is measured by Space Environment Viewing and Analysis Network (SEVAN) and by neutron monitor with 1-s resolution. The TGEs that persisted usually several minutes were mainly detected in the SEVAN channel 1 which has the lowest energy threshold, about 7-8 MeV. A statistical analysis shows that these enhancements usually occurred (not only) during large values of vertical, upward-pointing electric fields measured just above the detector. It is shown that the measurement of electric field at Skalnaté Pleso, distant about $1.86 \mathrm{~km}$ from the mountain top is also partly correlated with the enhancements and can provide additional useful information about the distance or dimension of charge structure and dynamics of electric field, especially on short time scales. The enhancements usually did not exceed several tens of percent of background values. However, events that exceeded the background values several times were also recorded. The most extreme event exceeded the background values about 215 times. This event was also detected by other SEVAN channels and by the neutron monitor ( $130 \%$ enhancement), which indicates a possibility of photonuclear reactions. The enhancements were often terminated by a nearby lightning.
\end{abstract}

Keywords: Thunderstorm ground enhancements of secondary cosmic ray flux, Gamma-ray glows, Neutron monitor, Electron acceleration, Particle detectors, Extreme event, Photonuclear reactions

\section{Introduction}

There are two distinct energetic phenomena in the Earth's atmosphere that generate gamma-rays during thunderstorms: terrestrial gamma-ray flashes (TGFs) and

\footnotetext{
${ }^{*}$ Correspondence: jachu@ufa.cas.cz

${ }^{1}$ Institute of Atmospheric Physics of the Czech Academy of Sciences,

Prague, Czech Republic

Full list of author information is available at the end of the article
}

gamma-ray glows. TGFs were discovered by Fishman et al. (1994) from satellite observations; they are short, usually less than $1 \mathrm{~ms}$ long, intense emissions that were initially associated with discharges in the mesosphere (sprites). Now, it is generally accepted that TGFs are produced at the tops of thunderclouds and are likely associated with intra-cloud (IC) lightning (Cummer et al. 2014; Dwyer and Uman 2014). On the other hand, gamma-ray 
glows - often called thunderstorm ground enhancements (TGEs), if observed on the ground (Chilingarian and Mkrtchyan 2012)-are less intense emissions of much longer duration, usually of several minutes. The enhancement of gamma-rays with respect to the background level is usually not larger than several $10 \%$ for most of the TGE events (Dwyer and Uman 2014; Kudela et al. 2017). However, TGEs that exceeded the background levels several times were also recorded (Chilingarian et al. 2010). Another difference from TGFs is that TGEs are not associated with individual lightning; on the contrary, they are terminated if a nearby lightning occurs (Lidvansky 2003; Tsuchiya et al. 2011; Kudela et al. 2017). TGEs are correlated with high values of electric fields and it is hypothesized that they are Bremsstrahlung from relativistic runaway electron avalanches (RREA), suggested by Gurevich et al. (1992). TGEs are usually observed on high mountain peaks (Torii et al. 2009; Tsuchiya et al. 2009; Chilingarian et al. 2011; Kudela et al. 2017) or at low altitudes during winter thunderstorms along the Japanese coast (Torii et al. 2002, 2011; Tsuchiya et al. 2011; Kuroda et al. 2016). The TGEs were also observed in the mountain valley at Baksan observatory, North Caucasus, Russia (Lidvansky 2003; Khaerdinov et al. 2005). These authors also mentioned an asymmetry of TGE observations with respect to the sign of the vertical component of electric field. Eack et al. (1996) and McCarthy and Parks (1985) observed gamma-ray glows from balloons and aircrafts.

Shah et al. (1985) were the first who reported an enhanced neutron flux associated with natural lightning. Other studies claiming detection of neutrons generated in thunderstorms followed (Martin and Alves 2010; Starodubtsev et al. 2012; Gurevich et al. 2012; Ishtiaq et al. 2016). Babich (2006) and Babich and Roussel-Dupré (2007) showed that photonuclear reactions are much more likely mechanism for the production of neutrons in thunderstorms than the previously assumed fusion of deuterium nuclei. Possible mechanisms of neutron production in thunderstorms, including electron-induced reactions, were discussed in detail by Babich et al. (2014) and Babich (2014). Enoto et al. (2017) observed TGEs with energies of $0.511 \mathrm{MeV}$ that followed lightning and lasted about $1 \mathrm{~min}$. Such energies are indicative for electron-positron annihilation. Enoto et al. (2017) showed that the positrons were likely produced by the decay of unstable isotopes of nitrogen 13 or oxygen 15 that can be created in the Earth's atmosphere by photonuclear reactions during intense TGFs that generated gamma photons of energies larger than $10.55 \mathrm{MeV}$. Neutrons are produced together with these unstable isotopes. The ground-based detection of neutrons, correlated with gamma-ray bursts, was recently also reported by Chilingarian et al. (2012a, b), Kuroda et al. (2016) and Bowers et al. (2017); in the latter case, the detection of neutrons was on subsecond scale, and was associated with TGF rather than with TGE.

Other gamma-ray emissions in the Earth's atmosphere with specific spectral lines can be caused by the decay of radon 222 daughter isotopes. These isotopes are usually washout by rain and might enhance the background level of gamma radiation during precipitations (Suszcynsky et al. 1996; Chilingarian 2018). The characteristic photon energies of such decay are below $3 \mathrm{MeV}$.

In this experimental paper, observations of enhanced secondary cosmic ray fluxes-TGEs by the detector system SEVAN (Chilingarian et al. 2018)-during measurements of large values of electric field at rocky mountain top of Lomnický Štít at the altitude of 2634 m in 2017 and 2018 are presented. The paper presents much stronger events compared to the previous study by Kudela et al. (2017) that was mainly based on statistical investigation of TGEs recorded by the SEVAN channel 1 in 2016. The analysis of individual strong TGE events, likely caused by RREA, is the main focus of the paper. An extreme TGE that exceeded the background values more than 200 times is presented. According to the best of authors' knowledge, this is likely the largest TGE that has been recorded by SEVAN. This event was also detected $(\sim 130 \%$ enhancement) by the neutron monitor located at Lomnický Štít (Kudela and Langer 2009). It is shown that the observations are consistent with photonuclear reactions. Moreover, electric field was measured not only at Lomnický Štít but also on its slope at the altitude of $1780 \mathrm{~m}$ at the observatory of Skalnaté Pleso since 2017. It is shown that distinct TGEs were observed for various values of electric field at these locations. Two-point (multi-point) measurements of electric field can provide useful information about the electric fields in thunderclouds during TGE observations and distances of generation region from observation point.

\section{Measurement setup}

The atmospheric electric field is measured by electric field mill EFM-100 sensors produced by Boltek company. The EFM-100 sensors are located at the rocky mountain peak of Lomnický štít (LS) and on its slope at Skalnaté Pleso (SP). Both sensors are mounted in inverted position to minimize precipitation noise. There are observatories with a necessary infrastructure at both locations. The horizontal and vertical distance between the two measuring sites is about 1650 and $854 \mathrm{~m}$, respectively (absolute distance about $1860 \mathrm{~m}$ ). Locations of the observatories are marked in the photograph shown in Fig. 1; the exact geographical coordinates and altitudes are listed in Table 1. The EFM-100 was installed at LS on 30 May 2016 and at SP about 1 year later, on 22 May 2017. The 


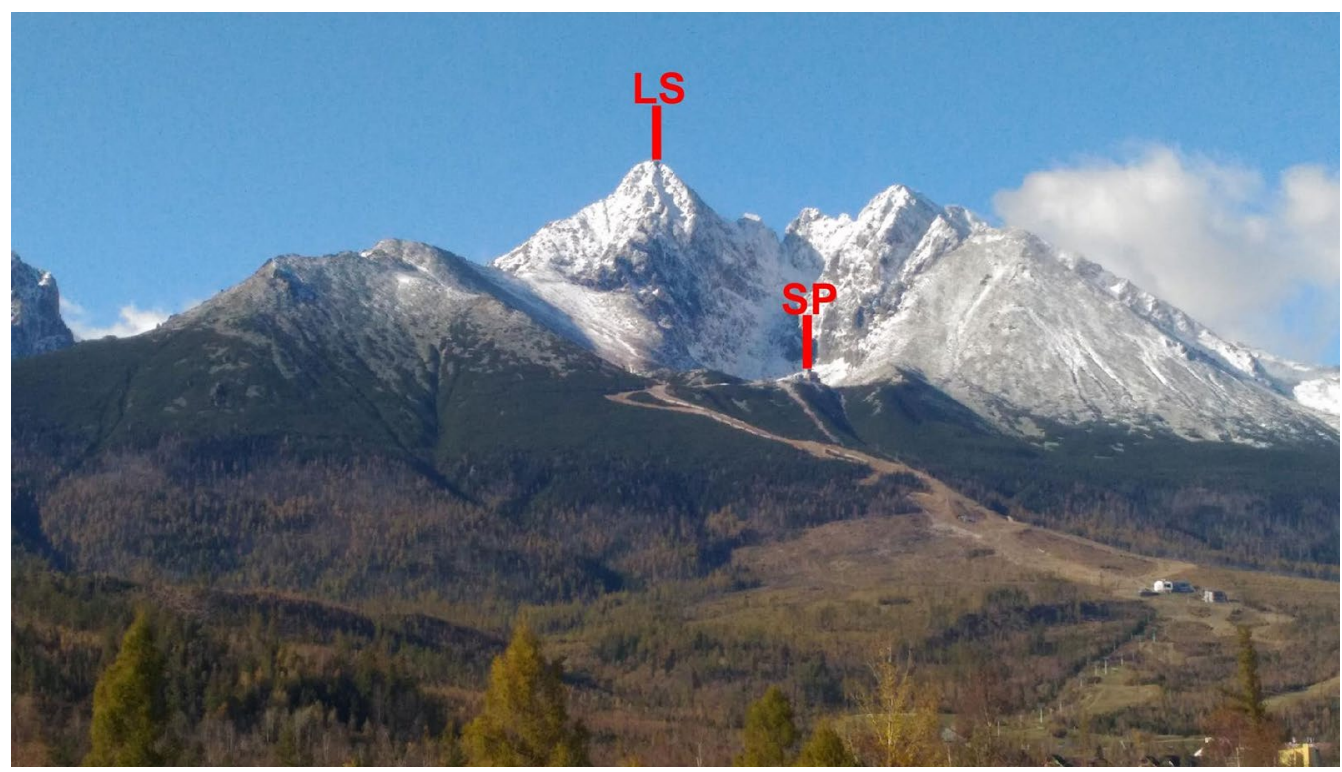

Fig. 1 View on Lomnický Štít with marked locations of observatories on its top (LS) and flank (SP)

Table 1 Geographical coordinates of measuring sites

\begin{tabular}{llll}
\hline Site & Latitude $\left(^{\circ}\right)$ & Longitude $\left(^{\circ}\right)$ & Altitude $(\mathbf{m})$ \\
\hline Lomnický štít (LS) & $49.1952 \mathrm{~N}$ & $20.2131 \mathrm{E}$ & 2634 \\
Skalnaté Pleso (SP) & $49.1894 \mathrm{~N}$ & $20.2340 \mathrm{E}$ & 1780 \\
\hline
\end{tabular}

EFM-100 located at the top of LS often suffers from icing in winter and it is therefore out of operation during winter to prevent damage of the rotor. The standard dynamic range of the EFM- 100 sensor is $\pm 20 \mathrm{kV} / \mathrm{m}$. The sensitivity of the sensor can be lowered using an external shunt resistor at the input amplifier following the EFM-100 manual, which results in a wider dynamic range. To prevent the saturation during thunderstorms, the dynamic range of the electric field measurement was set after short testing to $\pm 165 \mathrm{kV} / \mathrm{m}$ at LS and $\pm 40 \mathrm{kV} / \mathrm{m}$ at SP using proper external shunt resistors.

Secondary cosmic ray flux is measured at LS by detector system SEVAN (Chilingarian et al. 2018) and by neutron monitor with $1 \mathrm{~s}$ resolution. SEVAN and neutron monitor are located under the same roof that consists of two steel plates, $0.5 \mathrm{~mm}$ thick, separated by the $150-\mathrm{mm}$ thick layer of mineral wool.

SEVAN consists of three detectors (plastic scintillators). The upper and lower detectors are formed by 5 -cm-thick slabs with an area of $100 \times 100 \mathrm{~cm}^{2}$; they are separated by two 4.5 -cm-thick lead absorbers of the same area. The middle detector with dimensions of $50 \times 50 \times 25 \mathrm{~cm}$ is composed of five slabs and located between the two lead absorbers (Fig. 3 in
Chilingarian and Reymers 2008). Mainly the upper layer detector (channel 1) measures enhanced fluxes of secondary cosmic rays during thunderstorms. The estimated energy threshold of the channel 1 for low energy charged particles (electrons, muons) is about $3.6 \mathrm{MeV}$ (Chilingarian et al. 2015). Considering the roof above the SEVAN detector, the effective energy threshold for channel 1 is estimated to be 7-8 MeV (Kudela et al. 2017). The channel 1 is also partly sensitive to gamma-rays (Chilingarian et al. 2018). The middle detector (channel 2) mainly detects neutral particles (neutrons and gamma-rays). However, it is also partly sensitive to electrons with energies of several tens of $\mathrm{MeV}$. The lower detector (channel 3) detects high-energy charged particles (mainly muons) with energies exceeding 200-250 MeV (Chilingarian et al. 2018). Besides the counting of pulses in the individual scintillators, pulses that appeared simultaneously in different combinations of detector layers are also evaluated by the SEVAN electronics. In specific, the combinations $[1,1,0],[1,0,1],[0,1,1]$ and $[1,1,1]$ are implemented in hardware at LS. For example, counts for the $[1,1,0]$ combination mean that pulses occurred simultaneously in channels 1 and 2, but did not occur in channel 3. Other combinations can be calculated from the implemented measurements. For example, the combination $[0,1,0]$, which identifies counts that only appeared in channel 2, can be calculated by subtracting the counts in combinations $[1,1,0],[0,1,1]$ and $[1,1,1]$ from the counts recorded in channel 2 . The information obtained from combinations can be used to identify the 
type of incident particles; for example, the combination $[0,1,0]$ is indicative for neutral particles (Chilingarian and Reymers 2008).

The neutron monitor (NM), continually in operation since December 1981, is of the 8-NM-64 type and is also sensitive to low-energy neutrons (Kudela and Langer 2009). It is composed of eight SNM-15 counter tubes made in the former Soviet Union. The tubes have diameter of $15 \mathrm{~cm}$ and are $200 \mathrm{~cm}$ long. They are filled with $\mathrm{BF}_{3}$ gas in which the ${ }^{10} \mathrm{~B}$ isotopes represent at least $90 \%$ of the total boron. The centers of tubes are $50 \mathrm{~cm}$ apart. The counter tubes are surrounded by 2 -cm-thick cylindrical moderator from polyethylene and lead producer $(25 \mathrm{~cm}$ internal diameter, $35 \mathrm{~cm}$ external diameter, $205 \mathrm{~cm}$ long); all are covered by low-density polyethylene reflector $(405 \times 51 \times 225 \mathrm{~cm}$, the plates are $7.5 \mathrm{~cm}$ thick). According to a recent study by Aiemsa-ad et al. (2015), the NM64 is mainly sensitive to neutrons and protons. On the other hand, Tsuchiya et al. (2012) showed that even a small detection efficiency of neutron monitor to gamma-ray photons might cause a false detection of neutrons during thunderstorms as the gamma-rays are more abundant. In addition, Babich et al. (2013a, b) and Tsuchiya (2014) pointed out that the detected neutrons might not necessarily correspond to neutrons created in the atmosphere; the detected neutrons can also originate from photonuclear reactions that take place in the soil or materials surrounding the detector, e.g., the roof above the detector.

Lightning locations and times are obtained from the European lightning detection network EUCLID. The median location accuracy for cloud-to-ground (CG) discharges is about 100 to $200 \mathrm{~m}$. Location accuracy of EUCLID for intra-cloud (IC) discharges is undefined, as there is not a specific strike point and IC discharges may show significant horizontal extensions. The strike point coordinate of an IC discharge given by the EUCLID network results is somehow the center of field radiation from the IC discharge projected on the ground surface. The estimated detection efficiency is better than $98 \%$ for CG flashes and roughly between 70 and $80 \%$ for (IC) lightning (Chum et al. 2013). The detection efficiency is relatively low for upward lightning (Diendorfer et al. 2015), because upward lightning without any return strokes following the initial continuing current $\left(\mathrm{ICC}_{\text {only }}-\right.$ type flashes) is typically not detected.

\section{Data analysis and statistical results}

The measurements of electric field at LS and SP were calibrated using a reference EFM-100 that was located at several places with at least roughly flat surface around the permanently installed sensors. Despite of that the measured values should be considered as estimates because of complicated terrain (surface structure) which results in inhomogeneities of electric field, especially at LS, where the uncertainty was estimated to be around 30\% (Kudela et al. 2017). The sign of the measured electric field, output of the EFM-100, actually corresponds to a potential gradient (PG); $\mathrm{PG}=-E_{z}$, where $E_{z}$ is the vertical component of electric field. Positive values of PG represent downward-pointing electric fields. Positive values of PG are measured during fair weather conditions owing to a fair weather current flowing from the ionosphere to the ground; there is about $250 \mathrm{kV}$ potential difference between the ionosphere and the Earth's surface that is maintained by thunderclouds and other electrified clouds that act as batteries in the global electric circuit (Rycroft et al. 2000; Haldoupis et al. 2017). It should also be noted that the values of PG at LS and SP measured both at fair weather and during thunderstorms are much larger than those measured over flat terrain; PG is usually around $0.1 \mathrm{kV} / \mathrm{m}$ over flat terrain during fair weather (Rycroft et al. 2000). The median ( fair weather) values of PG are more than $2.8 \mathrm{kV} / \mathrm{m}$ at LS and about $0.17 \mathrm{kV} / \mathrm{m}$ at SP. During thunderstorms, the measured absolute values can exceed $140 \mathrm{kV} / \mathrm{m}$ at $\mathrm{LS}$ and $30 \mathrm{kV} / \mathrm{m}$ at $\mathrm{SP}$ as will be shown later. The minimum electric field $E_{\text {th }}$ needed for RREA mechanism to occur is $284 \mathrm{kV} / \mathrm{m} \times n_{\text {air }}$, where $n_{\text {air }}$ is the density of air relative to that at sea level at standard conditions (Dwyer and Uman 2014). Consequently, the thresholds $E_{\mathrm{th}}$ for LS and SP are 208 and $230 \mathrm{kV} / \mathrm{m}$, respectively. It should be noted that LS is a pointed rocky peak that is accessible with help of metallic chains fixed to the rock or by a cable car. Although the electric conductivity of the rocks containing only fractional amount of soil is low, it is still much larger than the conductivity of the air. Therefore, the LS attracts electric field lines as its summit is distinctly elevated above the surrounding. The staff at the observatory located at the top of LS often observes St. Elmo's fire (corona discharges) during thunderstorms. The LS is often inside the thunderclouds. Thus, the high values of PG (above $100 \mathrm{kV} / \mathrm{m}$ ) measured at LS seem reasonable.

To show the count enhancements in SEVAN channel 1 with respect to undisturbed background values, the residual counts are used. The residual counts were obtained as follows: first, the 1-min averages of 1-s counts obtained in channel $1, N_{\text {raw }}$, were corrected according to air pressure measured at LS as the measured counts partially depend on atmospheric pressure. Experimental relation $N_{\text {cor }}=N_{\text {raw }}$ .exp $[0.00432 \cdot(p-742.54)]$ was used to obtain counts $N_{\text {cor }}$ corrected (recalculated) to a mean pressure of $742.54 \mathrm{hPa}$ at LS, where $p$ is the measured air pressure in $\mathrm{hPa}$ and 0.00432 is the regression coefficient found by Kudela et al. (2017). Then, the background values were removed by subtracting the 6-h running median of $N_{\text {cor }}$ values 
from instantaneous $N_{\text {cor }}$ values to obtain residual counts, $N_{\text {res }}=N_{\text {cor }}-$ median $\left(N_{\text {cor }}\right)_{6 \mathrm{~h}}$. The median values of $N_{\text {cor }}$ were used instead of mean values because they are practically unaffected by significant count enhancements during the thunderstorms (events of interest) and represent the "quiet" background values better than the mean values. The residual values $N_{\text {res }}$ represent the fluctuations of $N_{\text {cor }}\left(N_{\text {raw }}\right)$ around their background values on short time scales. As the TGEs are usually several minutes or maximum tens of minutes long, 6-h medians represent well the undisturbed conditions and simultaneously take into account potential minor diurnal variations of $N_{\text {cor }}$ (Kudela et al. 2017).
Figure 2a shows the 1-min averages of 1-s $N_{\text {raw }}, N_{\text {cor }}$ and $N_{\text {res }}$ values by blue, green and red, respectively, recorded at LS from 1 May to 30 October 2018. Time $=0$ corresponds to the beginning of the analyzed period. The $N_{\text {raw }}$ values exhibit minor fluctuations associated with atmospheric pressure changes. However, the medians of $N_{\text {cor }}$ and $N_{\text {raw }}$ for the whole period of measurement differ by less than 1 count and are around 417 counts/s. The TGEs are observed in Fig. 2a as almost discrete local peaks. Note that the peaks of $N_{\text {res }}$ recorded on 24 May 2018 (day 23 in Fig. 2a) and on 19 October 2018 (day 171 in Fig. 2a) exceeded the scale in Fig. 2a. All the distinct events will be shown and discussed

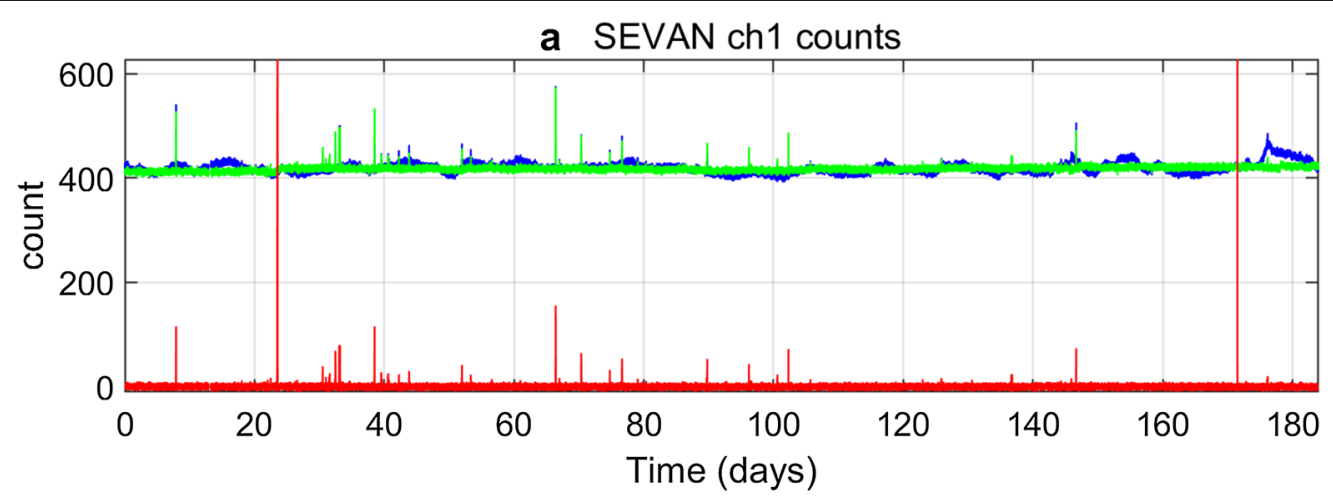

b Potential gradient at LS

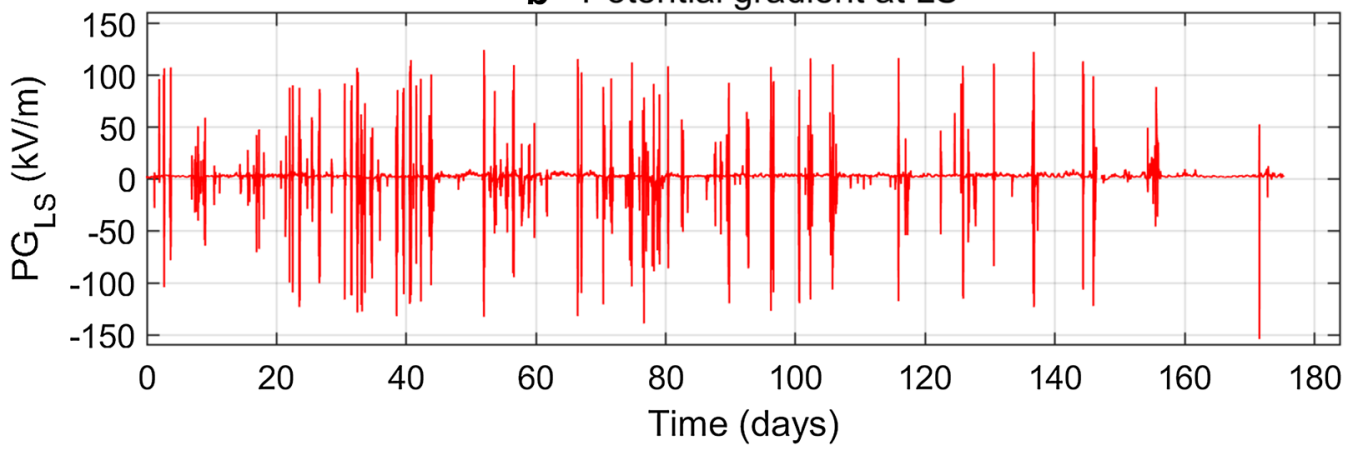

c Potential gradient at SP

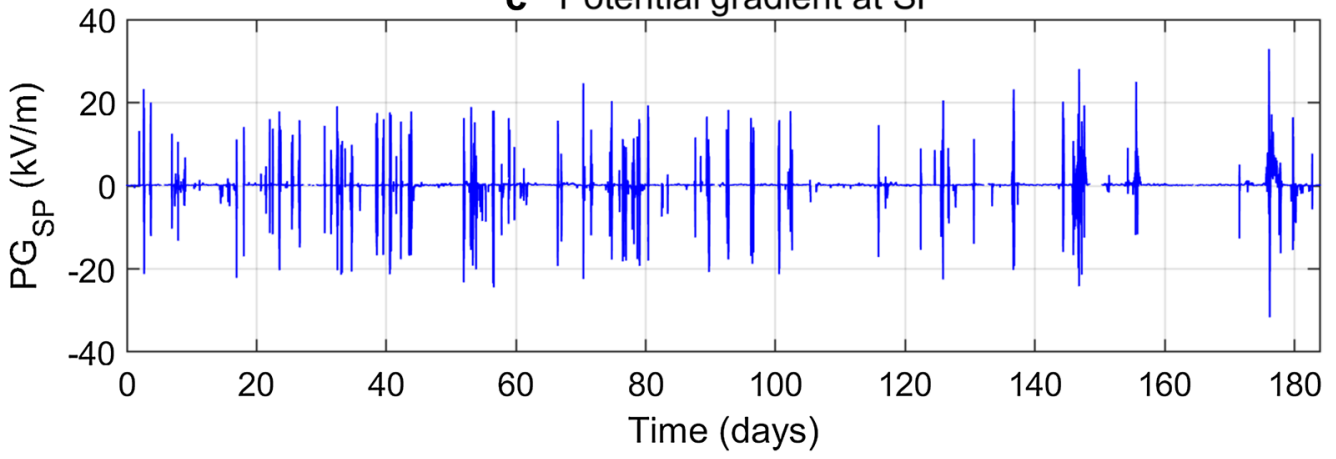

Fig. 2 Secondary cosmic rays and PG measured in 2018 from 1 May to 30 October. a 1-min averages of $N_{\text {raw }}$ counts in the SEVAN channel 1 (blue), counts $N_{\text {cor }}$ after correction on pressure (green), count enhancements $N_{\text {res }}$ with respect to background values (red). b PG measured at Lomnický peak. c PG measured at Skalnaté Pleso 
in detail in "Significant events" section. Figure 2b, c displays the PG measured at LS and SP, respectively. Because of icing, the measurements of PG at LS were terminated on 23 October 2018 to prevent a damage of the rotor of the EFM-100 sensor. The peaks of $N_{\text {res }}\left(N_{\text {raw }}, N_{\text {cor }}\right)$ correspond with the periods of enhanced PG, which will be shown in more detail in the next plots.

To visualize simultaneously PG measured at LS and SP in one plot (note the large differences between PG at LS and $\mathrm{SP}$ ) and to show their relative fluctuations, it is convenient to use normalized values of PG (normPG). The normalization is done by standard deviations $\sigma$ and with respect to median values separately for each location according to Eq. (1).

$$
\operatorname{normPG}=\frac{\mathrm{PG}-\operatorname{median}(\mathrm{PG})}{\sigma},
$$

Unlike mean values, the median values are almost not affected by large PGs during thunderstorms and represent relatively well the fair weather conditions. The standard deviations are simply calculated from the variance of all the measured data, including thunderstorm periods. The normalized PGs represent relative fluctuations of PG around the values recorded during fair weather conditions. The standard deviations used in the normalization are $11.36 \mathrm{kV} / \mathrm{m}$ and $1.82 \mathrm{kV} / \mathrm{m}$ for LS and SP, respectively; the median values are $2.83 \mathrm{kV} / \mathrm{m}$ and $0.173 \mathrm{kV} / \mathrm{m}$ at LS and SP, respectively.

Figure 3 presents histograms of significant enhancements of $N_{\text {res }}$ in dependence on PG measured at LS during the analyzed periods in 2017 and 2018 (from 23 May to 30 September 2017 and from 1 May to 23 October 2018). Normalized PG is used in Fig. 3. Note that normPG $\mathrm{LS}_{\mathrm{LS}}=1$ corresponds to the increase by $11.36 \mathrm{kV} / \mathrm{m}$ with respect to the typical fair weather value, which is about $2.83 \mathrm{kV} / \mathrm{m}$. Figure $3 \mathrm{a}$ shows number of minutes
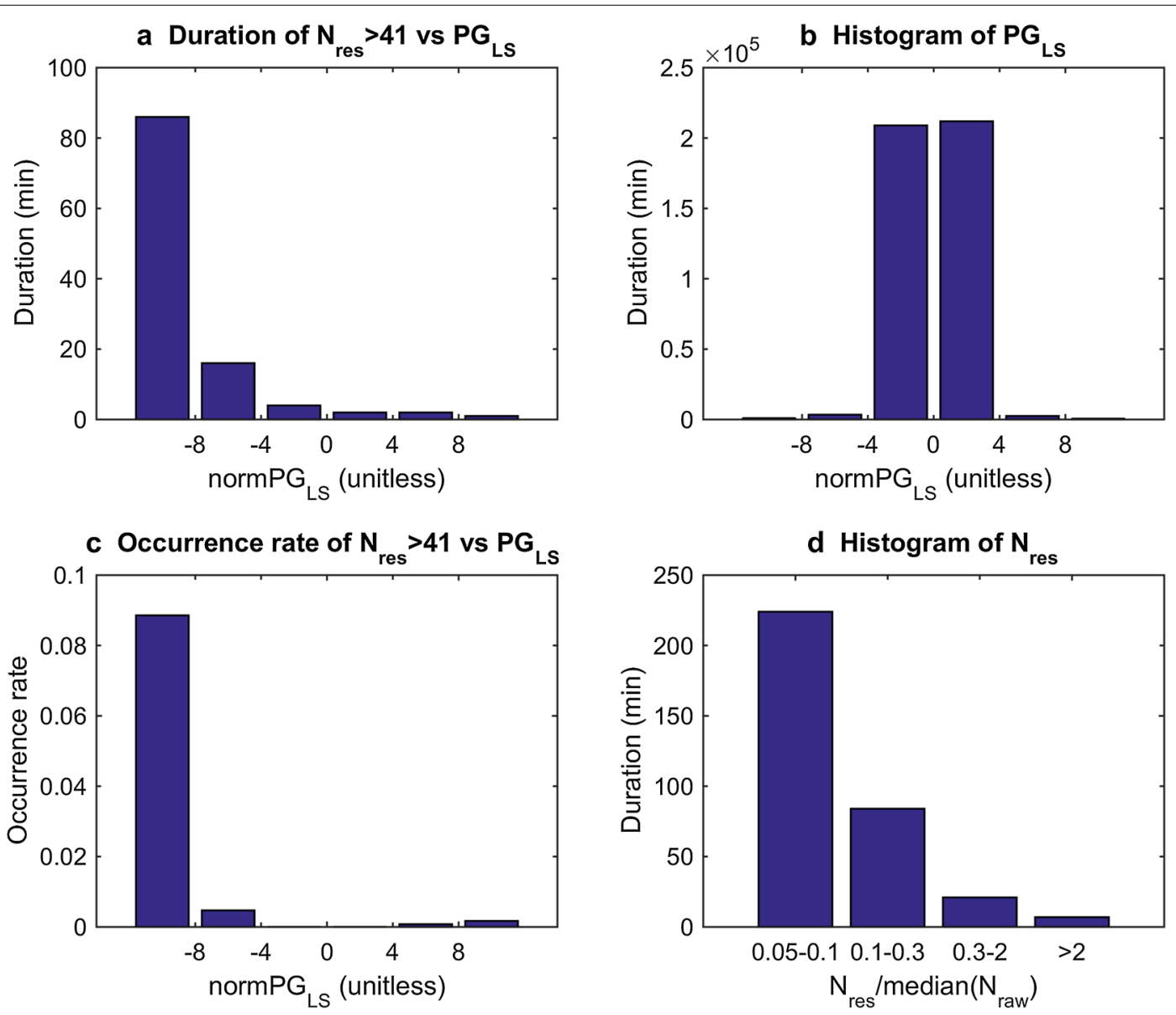

Fig. 3 Histograms summarizing the observation at LS. a Number of minutes when $N_{\text {res }}>41\left(N_{\text {raw }} / 10\right)$ for specific intervals of normalized PG. b Total duration of PG values in specific intervals. c Occurrence rate of $N_{\text {res }}>41\left(N_{\text {raw }} / 10\right)$ for various intervals of normalized PG. $\mathbf{d}$ Duration of enhancements in dependence on their relative values $N_{\text {res }} /$ median $\left(N_{\text {raw }}\right)$ 
when residual counts were larger than $41, N_{\text {res }}>41$. The total durations of such enhancements are presented separately for specific intervals (bins) of the normPG $\mathrm{LS}_{\mathrm{LS}}$. The bin width of 4 in normPG $\mathrm{LSS}_{\mathrm{LS}}$ corresponds to the step of about $45.4 \mathrm{kV} / \mathrm{m}$. The threshold $N_{\text {res }}=41$ represents about $10 \%$ enhancement with respect to background values $\left(N_{\text {res }} /\right.$ median $\left.\left(N_{\text {raw }}\right)=N_{\text {res }} / 417>0.1\right)$. It also corresponds to about two times the standard deviation $\sigma_{N}$ of 1-s background count values $N_{\text {raw }}$, provided the Poisson distribution, $\sigma_{N}=(417)^{1 / 2} \sim 20.4$. Figure 3a shows that the enhancements are more frequent for large negative values of PG rather than for large positive values of PG. To show that this result is not a consequence of possible higher occurrence rate of large negative PG, it is necessary to consider the duration of $N_{\text {res }}$ enhancement relative to duration of PG in the given intervals (bins). Figure $3 \mathrm{~b}$ presents the total duration that corresponds to the number of minutes when the measured normPG $\mathrm{G}_{\mathrm{LS}}$ was in the specific bins regardless of the occurrence of TGEs. Thus, the sum of durations over the given bins in Fig. 3b corresponds to the total length of the analyzed periods in 2017 and 2018 (about 10 months altogether). The occurrence rate of the enhancements that exceed $N_{\text {res }}>41$ is displayed in Fig. 3c, which shows the values shown in Fig. 3a normalized (divided) by the total duration of PG in the given bins that is presented in Fig. 3b. In other words, Fig. $3 \mathrm{c}$ shows the probability of significant $\left(N_{\text {res }}>41\right)$ enhancements, provided that the PG is in the given bin. Figure 3c shows that the occurrence rate of enhancements $\left(N_{\text {res }}>41\right)$ is highest for large negative values of $\mathrm{PG}_{\mathrm{LS}}$; it is much higher than for similar absolute values of positive $\mathrm{PG}_{\mathrm{LS}}$. We note that negative PG corresponds to upward-pointing electric field that accelerates electrons downward. Figure 3d displays a histogramduration of $N_{\text {res }}$ enhancements (in minutes) in dependence on the value of the relative enhancement. Obviously, the small enhancements are more frequent than the large ones.

It should be noted that the number of minutes when enhancements of $N_{\text {res }}$ were observed, shown in Fig. 3, does not correspond with the number of TGE events as the individual events usually took several minutes. Also, the $N_{\text {res }}$ values and electric field changed during the individual events. For example, only 6 events exceeded $50 \%$ of background counts $\left(N_{\text {res }} /\right.$ median $\left.\left(N_{\text {raw }}\right)>0.5\right)$. These significant individual events are presented in the next section.

\section{Significant events}

Significant TGEs characterized by $50 \%$ or higher enhancement of 1-s residual counts $N_{\text {res }}$ in SEVAN channel 1 with respect to background values $\left(N_{\text {res }} /\right.$ $\left.\operatorname{median}\left(N_{\text {raw }}\right)>0.5\right)$ are presented. First, events detected only by SEVAN channel 1 (estimated threshold 7-8 MeV) will be discussed, ordered according to their maximum values. Next, two TGEs detected also in SEVAN channels 2 and 3 will be shown. One of these TGEs is the strongest event that has been recorded at LS; it is also the only TGE that was detected by neutron monitor (NM).

\section{Significant TGEs detected only by SEVAN channel 1}

Figure 4a displays the TGE that reached about 50\% with respect to background values that occurred on 6 July 2018 around 10:02 UT. Large values of upward electric field were observed both at LS and SP (large negative values of normPG $\mathrm{PS}_{\mathrm{LS}}$ and normPG $\mathrm{SP}$ ) as documented in Fig. 4b. This corresponds to the usual situation for TGE observation as shown in Fig. 3a, c. The TGE was terminated by a discharge at 10:03:21.7 UT located by EUCLID at a distance of about $6 \mathrm{~km}$ from LS and classified as multiple stroke IC (Fig. 4c). The discharge was also detected by electric field measurement as a relatively large sudden increase-upward step of PG recorded both at LS and SP (Fig. 4b) at the time of the TGE termination. Another point worth mentioning is that the TGE shown in Fig. 4a more or less reached its maximum at the time of its termination by lightning. This is different from the TGE that occurred on 29 June 2017 around 15:53 UT, shown in Fig. 5. This TGE grew rapidly until 15:54:05 UT when it was terminated by an IC discharge with peak current of $\sim+11 \mathrm{kA}$ located by EUCLID at LS (Fig. 5c); more precisely, the IC discharge that terminated the TGE was likely a part of the multiple stroke discharge. The peak current and polarity of discharges detected by EUCLID are color-coded in all the plots presenting lightning locations. Red colors correspond to positive (downward) currents, whereas blue colors represent negative (upward) currents; the darker the color, the larger was the current of the detected discharge. A logarithmic function $\operatorname{sign}(I) \cdot \log _{10}(1+I)$ was applied to visualize the wide range of lightning peak currents simultaneously with their sign, where $I$ is the peak current in kA and $\operatorname{sign}(I)$ is $1(-1)$ for positive (negative) discharges. The lightning is also detected as a sudden decrease-downward step in electric field (PG) measured at LS; it was not clearly detected in electric field measured at SP (Fig. 5b), where only a minor upward step was recorded. The TGE reached about $100 \%$ of the background values $\left(N_{\text {res }}\right.$ was over 400 ), and there was an obvious tendency of the TGE to increase at the time of termination. There was mostly downward electric field (positive PG) both at LS and SP when the TGE was recorded, which is an infrequent situation.

Figure 6 shows the TGE recorded on 24 May 2018 around 12:58 UT. This TGE reached about $250 \%$ of the background values. After reaching its maximum, the 
a Counts on 2018-07-06

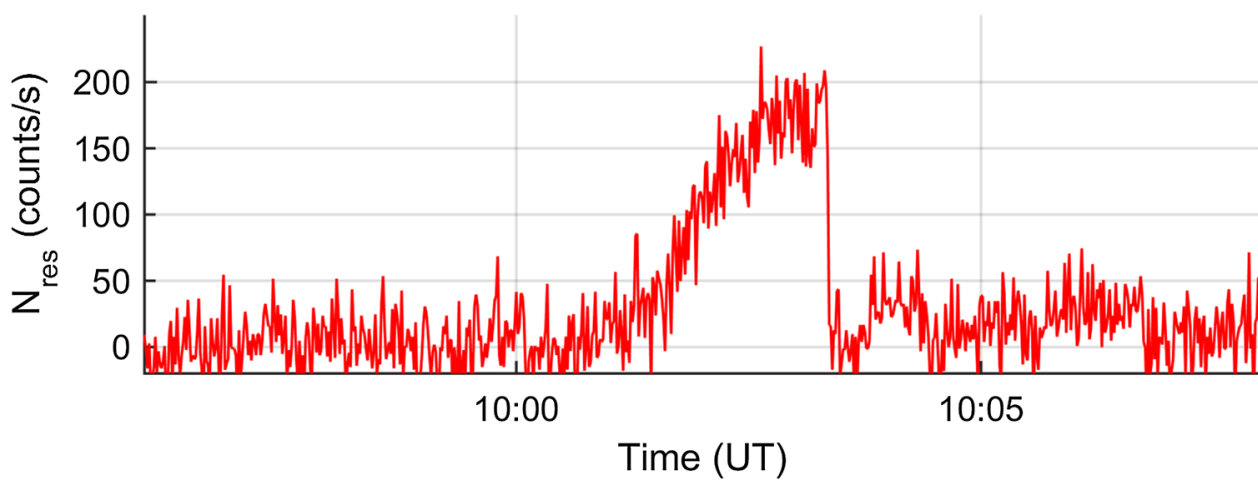

b Normalized PG on 2018-07-06

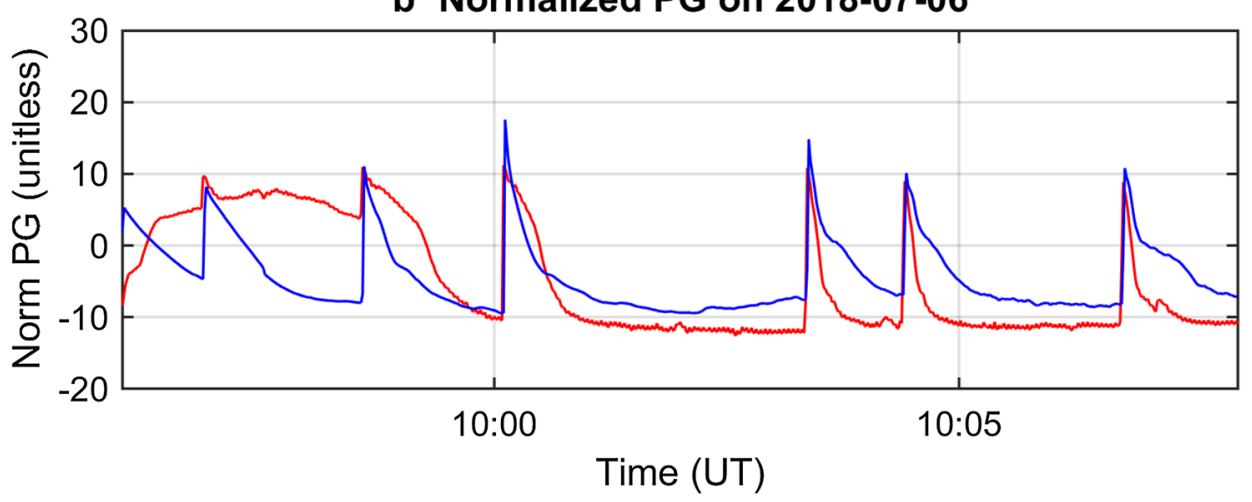

C Lightning Distance and color coded sign $(I)^{*} \log _{10}(1+1[k A])$

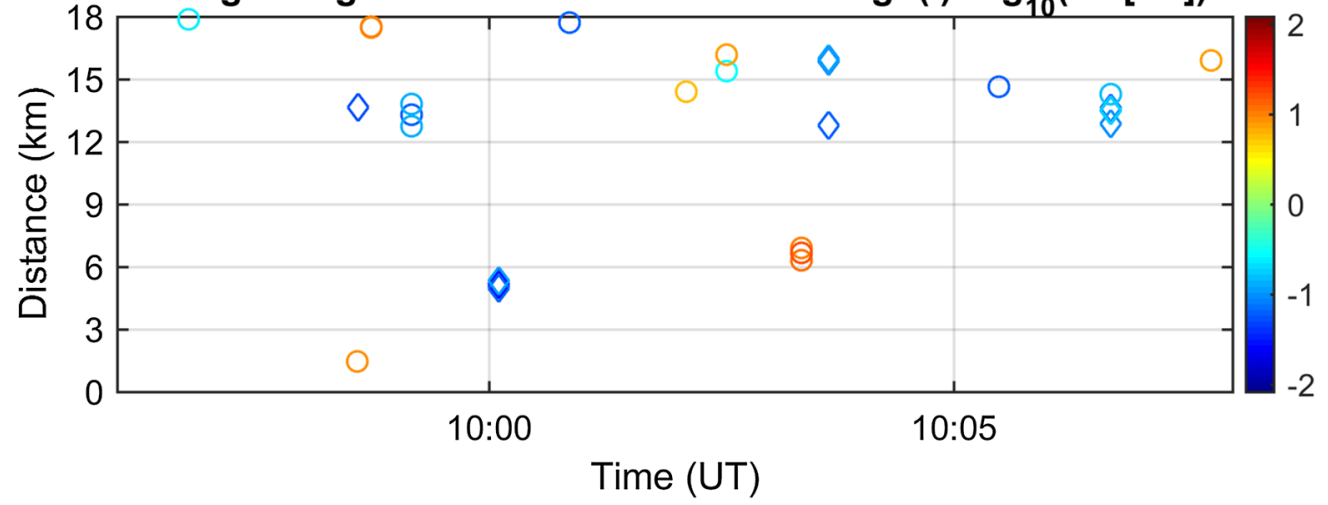

Fig. 4 Event recorded on 6 July 2018 around 10:02 UT. a Residual counts at Lomnicky peak by red. b Normalized PG at Lomnicky peak (red) and Skalnate Pleso (blue). c Lightning distances from Lomnicky peak as recorded by EUCLID lightning detection network; CG discharges are by diamonds, IC by circles, peak current / is color-coded; the color approximately corresponds to the common logarithm of the peak current in KA, sign of the current is distinguished

TGE spontaneously decayed. However, before reaching its background values, it was terminated by a multiple stroke discharge with dominant CG stroke with peak current of $+38 \mathrm{kA}$ at 13:00:34 UT located by EUCLID at the distance of $\sim 2.8 \mathrm{~km}$ from LS. The discharge can be clearly seen as a sudden increase (step) in electric field data (PG) at SP and just minor step at LS. That shows that a discharge at a distance of $\sim 3 \mathrm{~km}$ is responsible for the charge removal and reduction of electric field in the thundercloud, which resulted in the termination of nearby TGE or that the TGE originated from a relatively large distance $(\sim 1 \mathrm{~km}$ or more). The electric field had large values and the expected upward orientation at SP; however, both the values and orientation of electric field 


\section{a Counts on 2017-06-29}

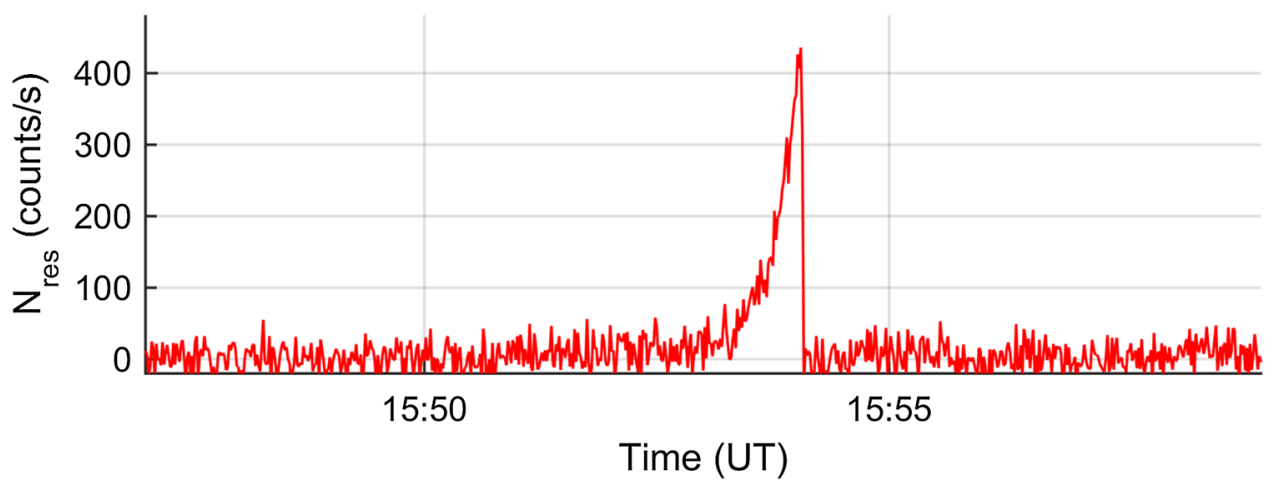

b Normalized PG on 2017-06-29
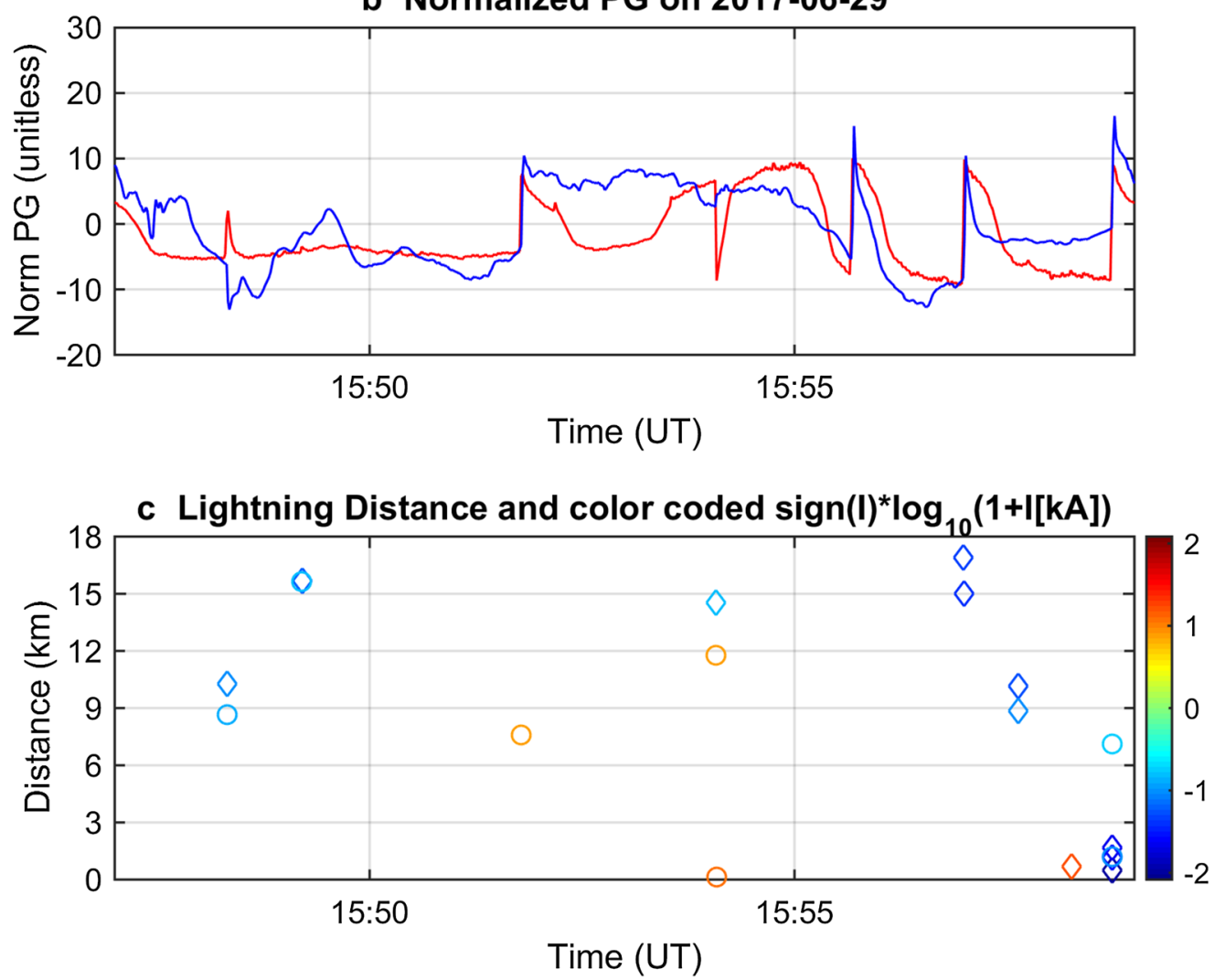

Fig. 5 Event recorded on 29 June 2017 around 15:53 UT. a Residual counts at Lomnicky peak by red. b Normalized PG at Lomnicky peak (red) and Skalnate Pleso (blue). c Lightning distances from Lomnicky peak as recorded by EUCLID lightning detection network; CG discharges are by diamonds, IC by circles, peak current / is color-coded; the color approximately corresponds to the common logarithm of the peak current in KA, sign of the current is distinguished

fluctuated at LS during the event (Fig. 6b), which supports the latter possibility.

Figure 7 presents a strong TGE that exceeded the background values about 15 times $\left(N_{\text {res }} /\right.$ median $\left.\left(N_{\text {raw }}\right) \sim 15\right)$. The event was recorded after the summer thunderstorm season, in the autumn on 19 October 2018 around 12:14 UT. This TGE lasted nearly $5 \mathrm{~min}$ and was not terminated by any lightning. Its duration was likely given by the passage of the charged cloud over the LS. The electric field at LS reached extreme values with upward orientation (normPG - 14, PG - $160 \mathrm{kV} / \mathrm{m}$ ) during the event. The electric field at SP had the same orientation; however, the values were moderately large, not extreme. A possible interpretation is that LS was inside the thundercloud, 


\section{a Counts on 2018-05-24}

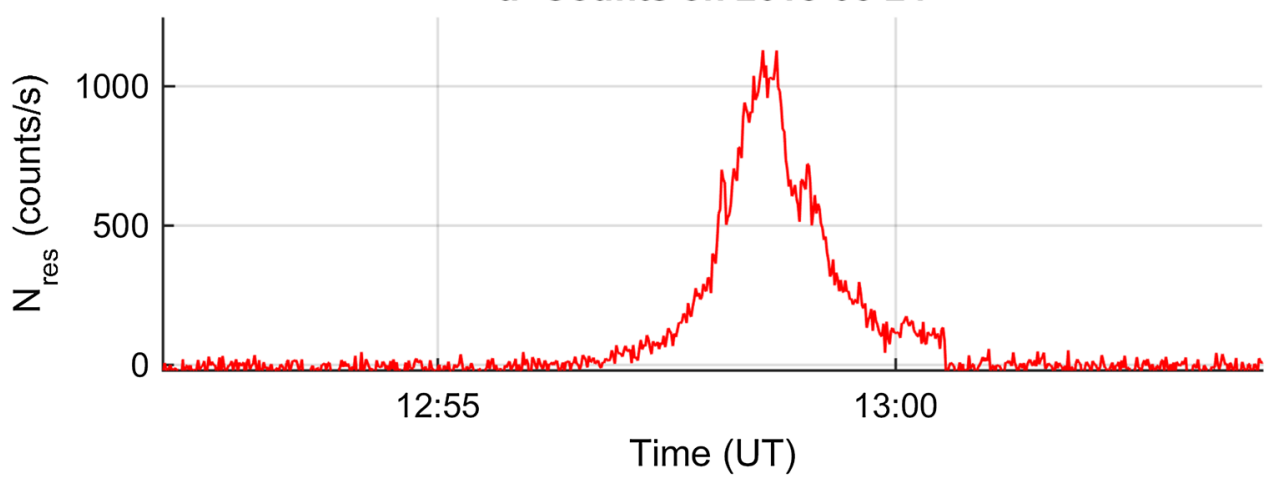

b Normalized PG on 2018-05-24

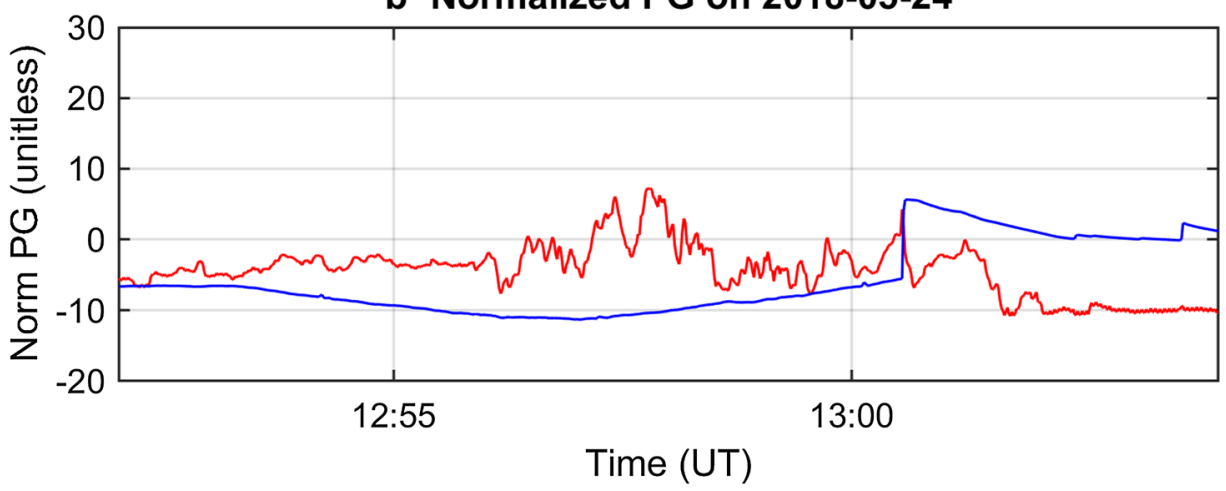

C Lightning Distance and color coded sign $(I)^{*} \log _{10}(1+I[k A])$

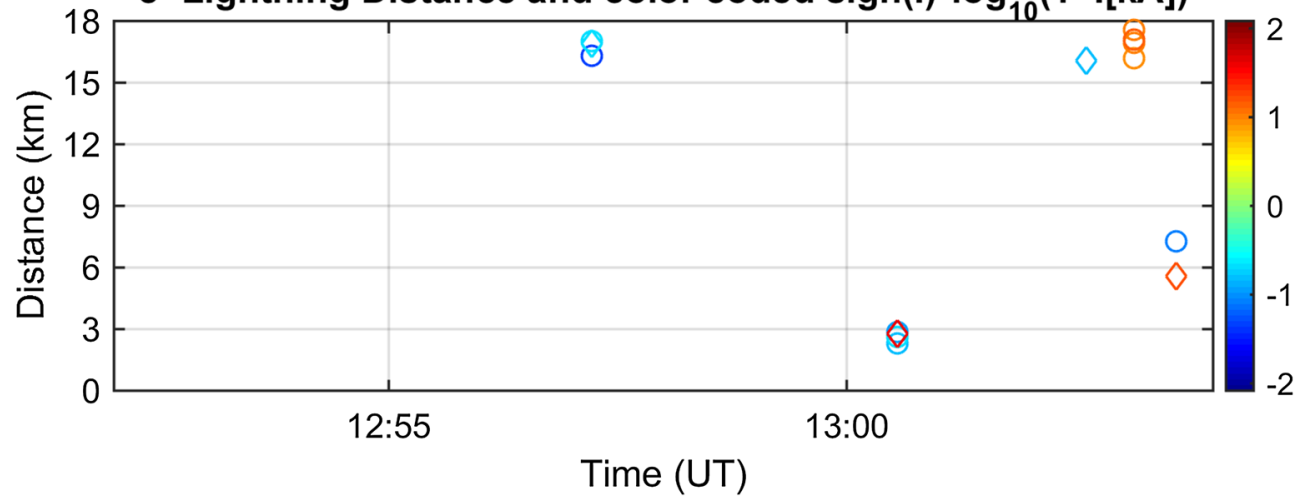

Fig. 6 Event recorded on 24 May 2018 around 12:58 UT. a Residual counts at Lomnicky peak by red. b Normalized PG at Lomnicky peak (red) and Skalnate Pleso (blue). c Lightning distances from Lomnicky peak as recorded by EUCLID lightning detection network; CG discharges are by diamonds, IC by circles, peak current / is color-coded; the color approximately corresponds to the common logarithm of the peak current in KA, sign of the current is distinguished

close to negative charge center and likely also close to origin of the TGE.

\section{TGEs detected in all SEVAN channels}

Figure 8 displays TGE that occurred on 30 May 2017 around 14:24 UT and was recorded by all three SEVAN channels. Figure 8 has the same format as Figs. 4, 5, 6,
7 and shows residual counts $N_{\text {res }}$ from SEVAN channel 1, electric field and lightning locations by EUCLID. Raw counts recorded in SEVAN channels 1, 2 and 3 are shown in Fig. 9a. In addition, the coincidences described in "Measurement setup" are displayed in Fig. 9b. Figure 9c presents the raw counts of NM; no enhancement was observed in this case by NM. The TGE recorded in 


\section{a Counts on 2018-10-19}

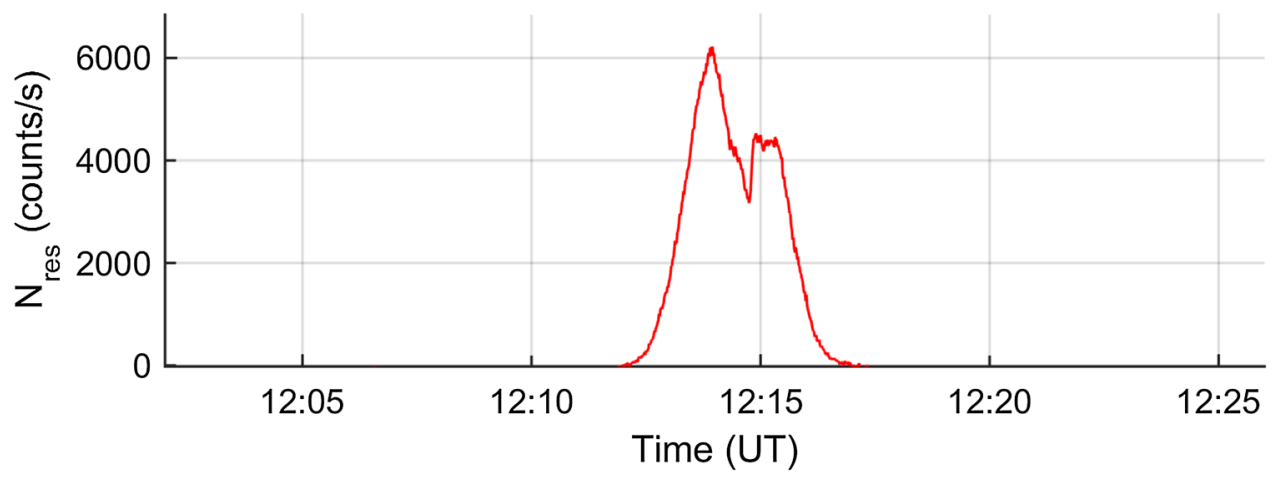

b Normalized PG on 2018-10-19

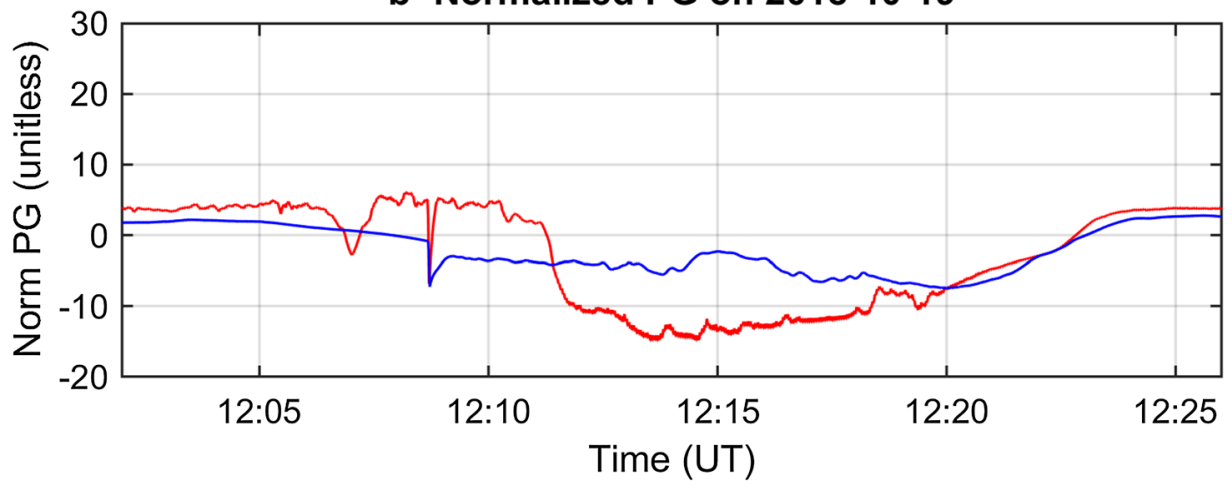

\section{c Lightning Distance and color coded sign $(1)^{\star} \log _{10}(1+[[k A])$}

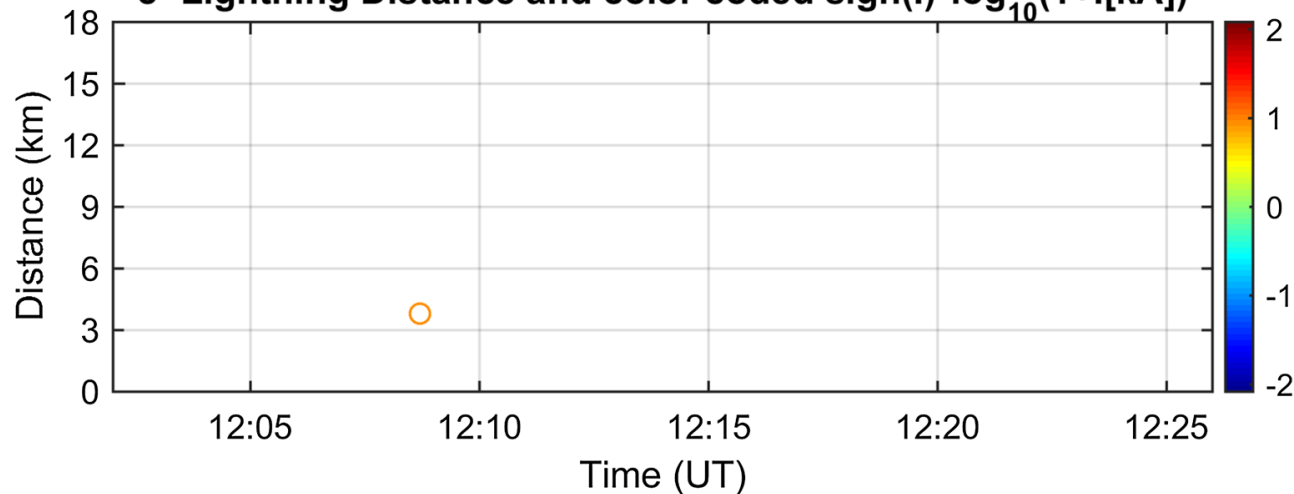

Fig. 7 Strong event recorded on 19 October 2018 around 12:14 UT. a Residual counts at Lomnicky peak by red. b Normalized PG at Lomnicky peak (red) and Skalnate Pleso (blue). c Lightning distances from Lomnicky peak as recorded by EUCLID lightning detection network; CG discharges are by diamonds, IC by circles, peak current / is color-coded; the color approximately corresponds to the common logarithm of the peak current in KA, sign of the current is distinguished

SEVAN channel 1 (Fig. 8a) does not seem extraordinary in terms of amplitude; the enhancement did not exceed $100 \%$ of background values. It is a relatively long event that lasted nearly $15 \mathrm{~min}$. It was two times interrupted by IC discharges at 14:17:17 UT and at 14:27:08 UT. Both lightning were detected by EUCLID (Fig. 8c) and by electric field measurement at LS (Fig. 8b). There was a data gap in electric field data at $\mathrm{SP}$, and therefore normPG $\mathrm{SP}_{\mathrm{SP}}$ is not displayed. Remarkable spikes in $N_{\text {res }}$ were observed at times of these discharges. Figure 9 shows that these spikes are visible in all three SEVAN channels and in the coincidence $[1,1,1]$ that corresponds to simultaneous occurrence of signal in all three channels. The spikes are likely caused by the electromagnetic interference 


\section{a Counts on 2017-05-30}

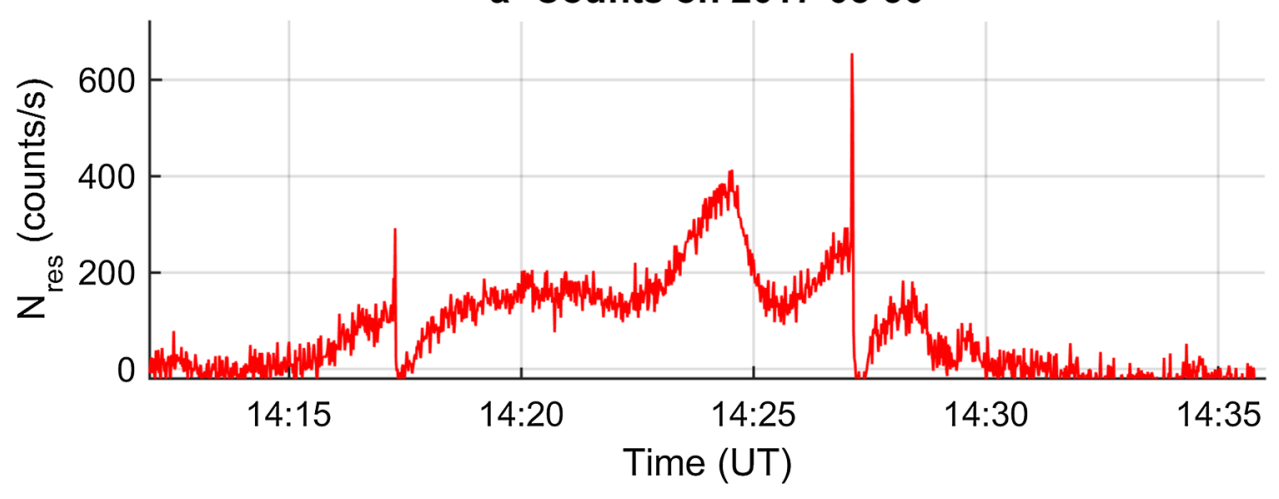

b Normalized PG on 2017-05-30
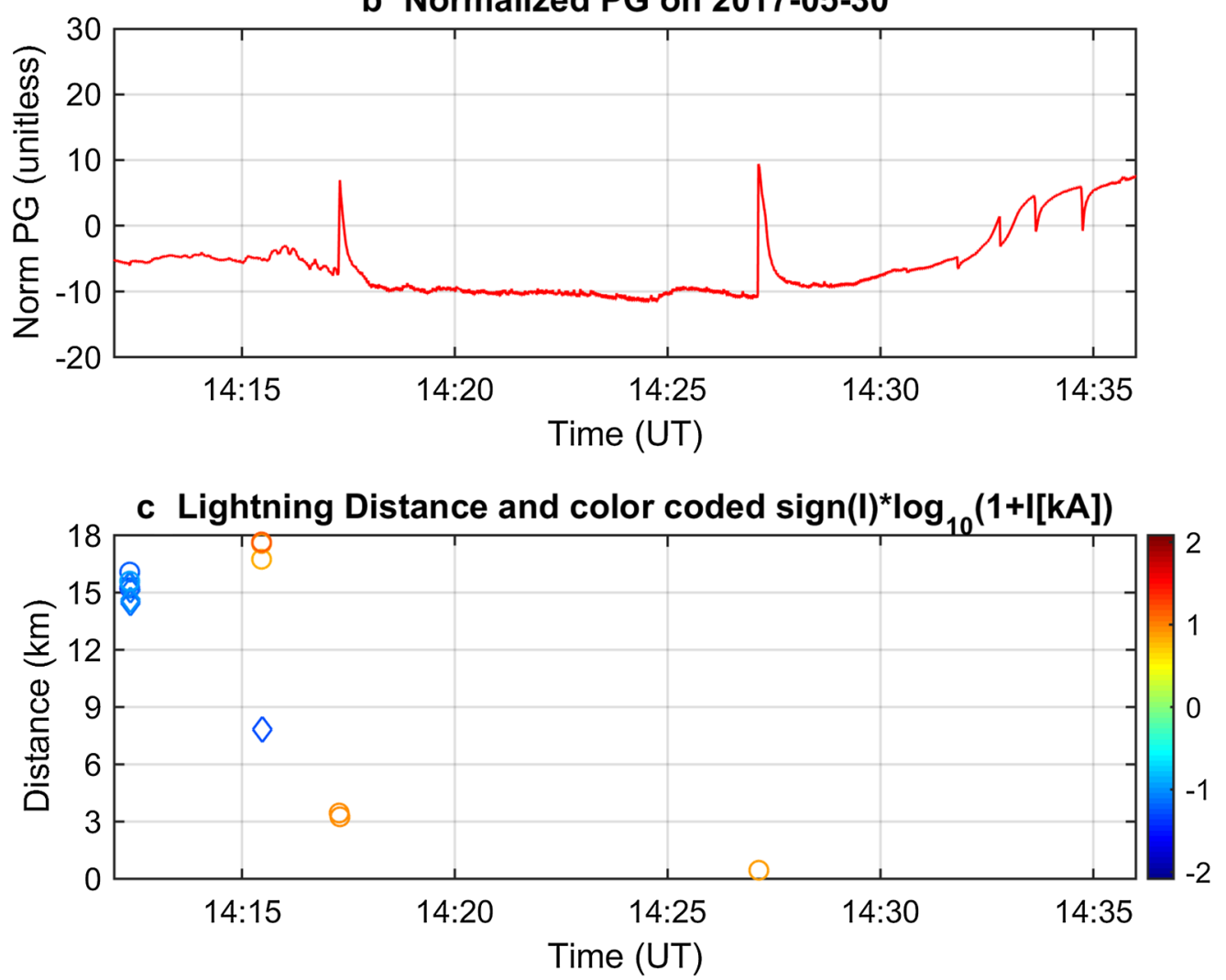

Fig. 8 Event recorded on 30 May 2018 around 14:24 UT. a residual counts at Lomnicky peak by red. b Normalized PG at Lomnicky peak (red). c Lightning distances from Lomnicky peak as recorded by EUCLID lightning detection network; CG discharges are by diamonds, IC by circles, peak current / is color-coded; the color approximately corresponds to the common logarithm of the peak current in kA, sign of the current is distinguished

(impulse) from the discharges. On the other hand, the count enhancements that persist about $15 \mathrm{~min}$ in all three channels and have roughly the same shape and amplitude, if the offsets (background values) are removed (Fig. 9a), are difficult to explain by electromagnetic interference from lightning or from local corona discharges. Moreover, a signal of similar shape and amplitude as in channels 1,2 , and 3 is observed in the coincidence $[1,1,1]$. Such a situation has not been observed for other events even for larger electric fields at LS. A possible explanation for that is (provided that it is not an unknown, strange instrumental effect) that particle flux did not pass through the lead layers separating the individual SEVAN detectors and arrived on SEVAN roughly horizontally 


\section{a SEVAN channels on $2017-05-30$}

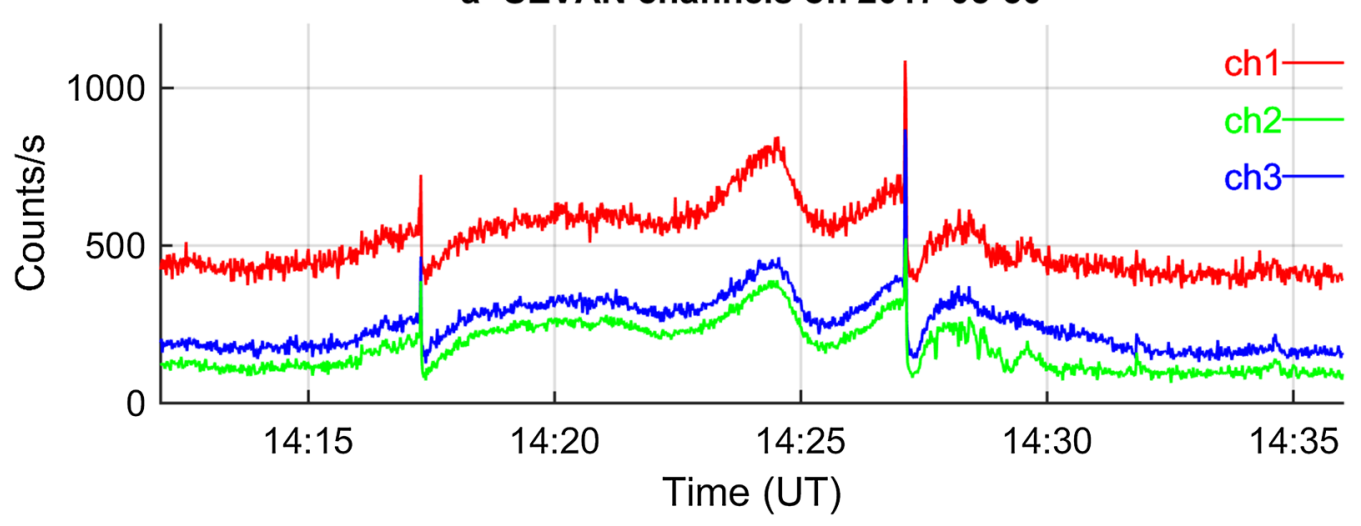

b SEVAN coincidences on 2017-05-30

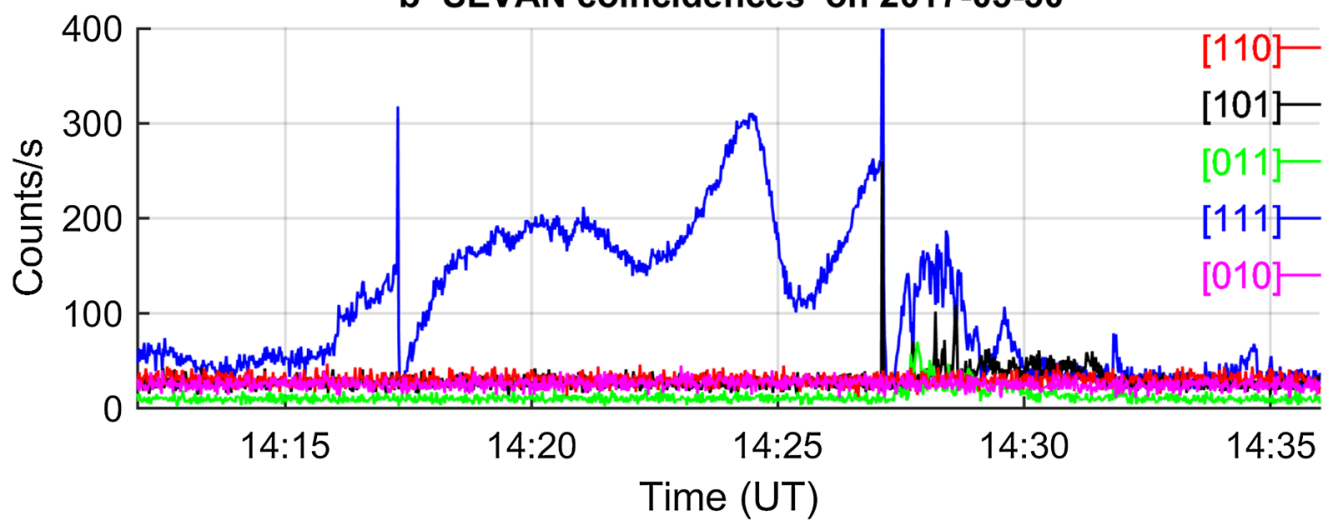

c Neutron monitor on 2017-05-30

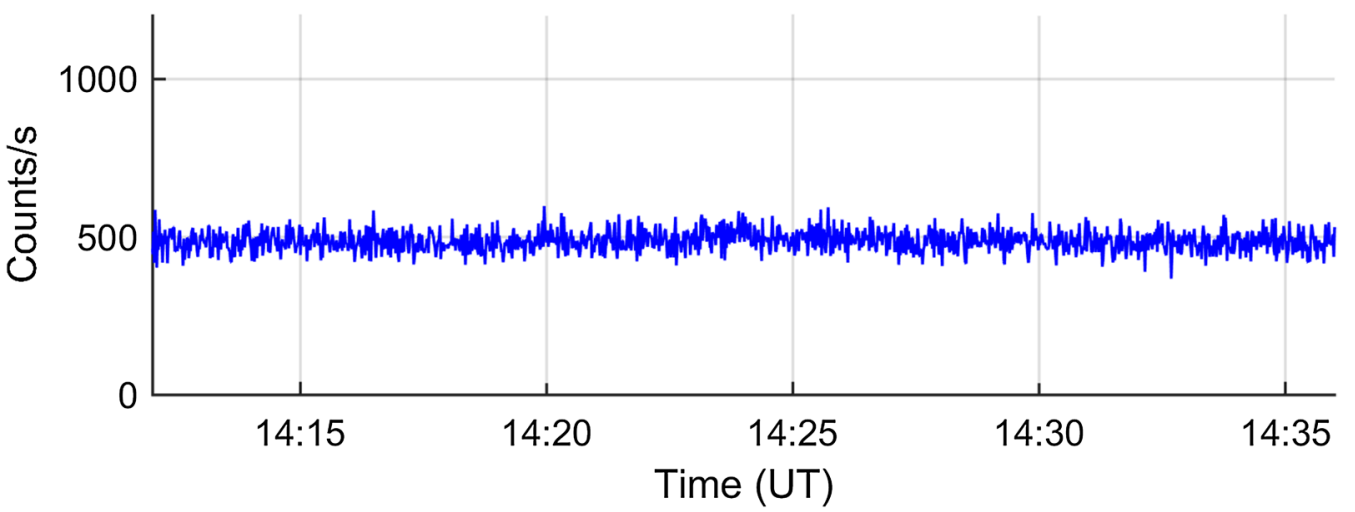

Fig. 9 Event recorded by SEVAN and neutron monitor on 30 May 2017 around 14:24 UT. a Raw counts recorded by individual SEVAN channels. b Coincidences recorded by different combinations of SEVAN channels. c Raw counts recorded by neutron monitor

from side. Note that if the particles pass through the lead layers (vertical or quasi-vertical angle of incidence), then various channels (layers) detect particles of different energies. However, no screening by lead layers takes place if the particle flux arrives on SEVAN horizontally from side. Then, all the channels experience approximately the same energy threshold. The possibility of side arrival is also supported by the lack of significant signals in other coincidences that would be indicative for specific energies or particles. Thus, it is hypothesized that this particular TGE could be caused by gamma-rays or electrons of similar energies as the TGEs presented in Figs. 4, $5,6,7$; the only difference was the angle of incidence on the detector. Unfortunately, the data gap in the electric 
field measurement at SP does not allow to check if the configuration of electric field was different than for other events.

Figure 10a displays the strongest event that was recorded on 10 June 2017 around 13:14 UT.

The residual counts $N_{\text {res }}$ reached $\sim 90,000$ before the TGE termination by multi-stroke discharge at 13:14:35.5
UT. The peak values in SEVAN channel 1 exceeded the background values about 215 times. Several strokes of this complicated discharge were located by EUCLID to the summit of LS (observatory) or to its close vicinity (Fig. 10c). This is a likely reason for a short data gap in electric field measurement at LS starting at the time of this discharge. Nevertheless, the discharge is well visible

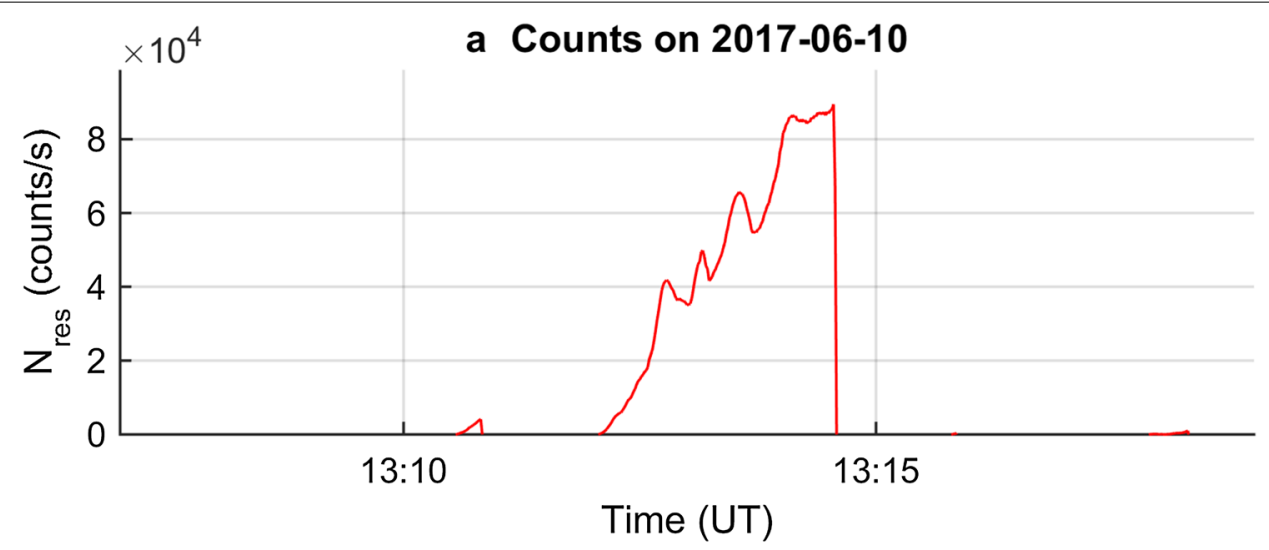

b Normalized PG on 2017-06-10

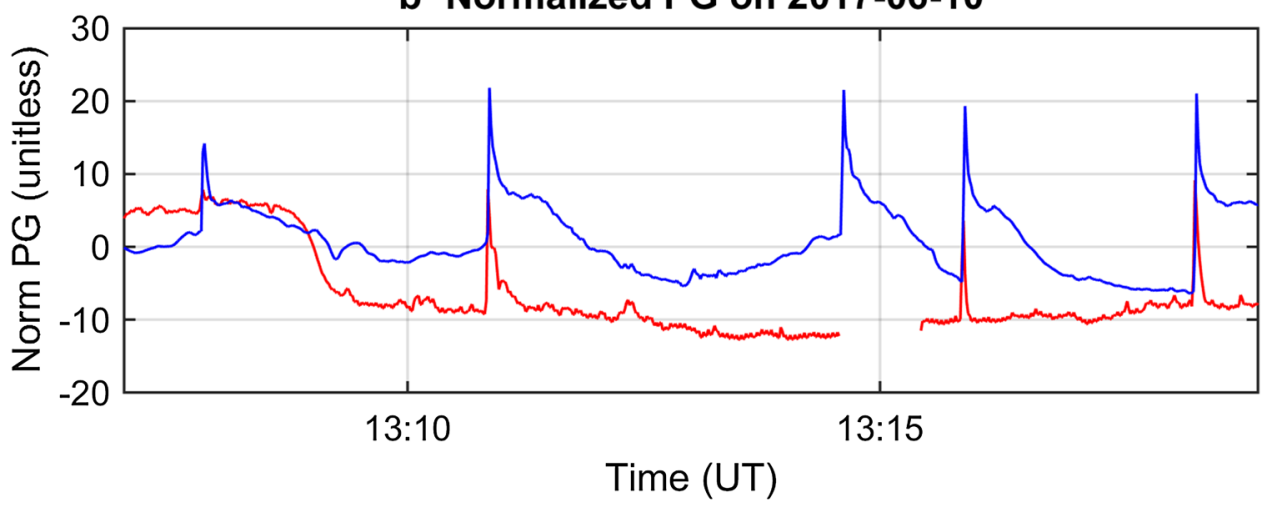

c Lightning Distance and color coded sign $(I)^{*} \log _{10}(1+\mid[k A])$

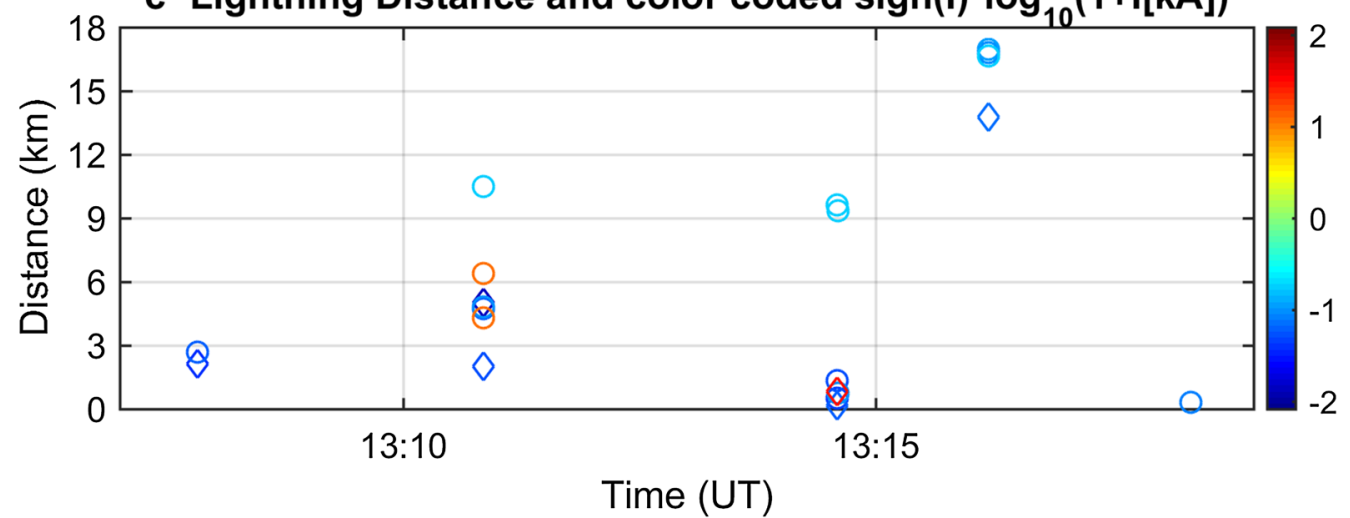

Fig. 10 Extreme event recorded on 10 June 2017 around 13:13 UT. a Residual counts at Lomnicky peak by red. b Normalized PG at Lomnicky peak (red) and Skalnate Pleso (blue). c Lightning distances from Lomnicky peak as recorded by EUCLID lightning detection network; CG discharges are by diamonds, IC by circles, peak current / is color-coded; the color approximately corresponds to the common logarithm of the peak current in kA, sign of the current is distinguished 
as an upward step in the electric field data recorded at SP (Fig. 10b). Large values of upward electric field reaching $140 \mathrm{kV} / \mathrm{m}$ were observed at LS during the TGE, whereas moderate values of electric field were recorded at SP. Figure 11 shows raw data from all three SEVAN channels, including their coincidences, and data from NM. The counts in channel 1 were divided by 1000 to fit in the plot (Fig. 11a). The count enhancements are observed in all three channels. However, the amplitudes of these enhancements were different because of various sensitivities to different particles and energies. Considering peak values, the enhancements were $\sim 90,000, \sim 100$ and $\sim 200$ in channels 1, 2 and 3, respectively. Importantly, the count enhancement of about 50-60 is observed for

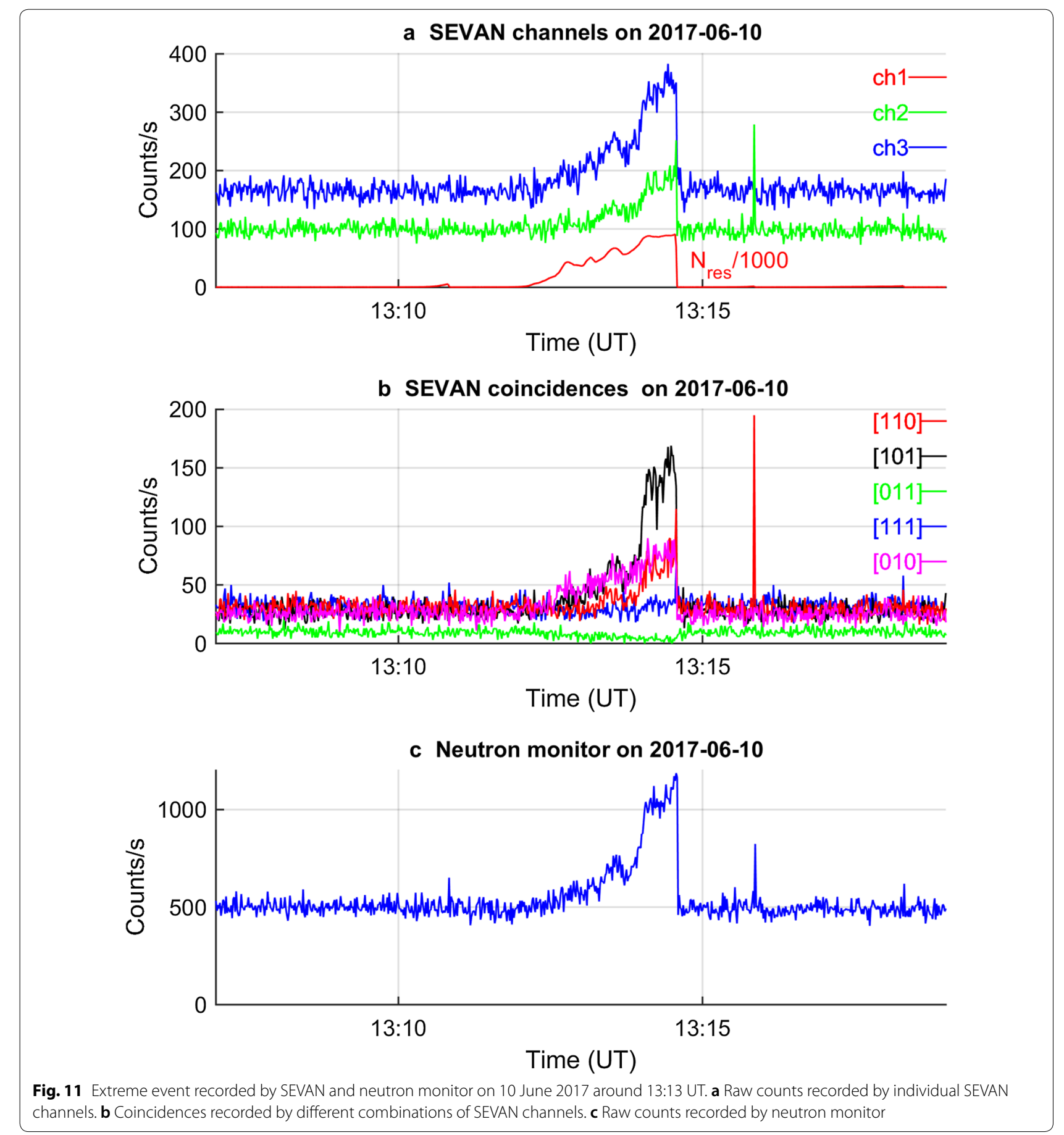


combination $[0,1,0]$, which indicates neutrons or high energetic gamma-rays (Chilingarian et al. 2018). The enhancement in the coincidences $[1,1,0]$ and $[1,0,1]$ indicates presence of high-energy charged particles with energies that may exceed $\sim 200 \mathrm{MeV}$ for the $[1,0,1]$ coincidence (Chilingarian and Reymers 2008). We consider that enhancement of high-energy electrons is observed in this case as the combination $[1,1,1]$ that is indicative for detection of muons (Chilingarian et al. 2018) does not show any enhancement. In addition, previous studies and observations (e.g., Hariharan et al. 2019 and references therein) show that total cosmic ray muon flux might be partly attenuated by large atmospheric electric fields. Indeed, a careful inspection, based on 1-min running averages of the signal shown in Fig. 11b, reveals that the combination $[1,1,1]$ exhibits a minor decrease during the event (a more obvious minor decrease in $[0,1,1]$ combination is likely caused by the quasi-saturation of channel 1 as it is difficult to find time intervals, when no particle was detected in channel 1). The detected electron energies and hence also energies of subsequent Bremsstrahlung are consistent with photonuclear reactions that can occur in the atmosphere (Enoto et al. 2017) or in the material surrounding the detector (Tsuchiya 2014). The possibility of neutron detection is also supported by the count enhancement, up to about $130 \%$ with respect to background values, in NM (Fig. 11c). As noted by Tsuchiya et al. (2012), the neutron monitor is also partially sensitive to gamma-rays with energies higher than $\sim 7 \mathrm{MeV}$; the sensitivity increases with gammaray energy. Thus, the signal in NM is likely caused by a mixed field of gamma-rays and neutrons. The spike observed at 13:15:52 UT in ch2, coincidence $[1,1,0]$ and $\mathrm{NM}$ about $77 \mathrm{~s}$ after the termination of the TGE is most likely caused by electromagnetic impulse from lightning. An alternative, rather speculative explanation could be a detection of TGF. The 1-s time resolution does not allow to distinguish between these two possibilities.

\section{Discussion}

It was shown that the enhancements of secondary cosmic ray flux (TGEs) represented by increases of $N_{\text {res }}$ in SEVAN channel 1 usually occurred during large values of upward-pointing electric field (negative PG), which means that electrons were accelerated downward. This relation is more obvious for LS rather than for SP. It should be noted that the extreme value of electric field at LS does not automatically mean a strong TGE, which is documented by the occurrence rates presented in Fig. 3c. The relation between electric field and $N_{\text {res }}$ is also obvious from scatterplots in Fig. 12a-f that show in an alternative way the analyzed significant events. Figure $12 \mathrm{a}, \mathrm{d}$, e, f corresponds to cases when TGE ( $N_{\text {res }}$ enhancement) occurred if PG at LS (red) reached large negative values. This is a typical situation, observed also at mount Aragats, Armenia (Chilingarian, 2014). On the other hand, Fig. 12b presents TGE that occurred at large positive PG (downward-pointing electric field) on 29 June 2017. Figure 12c shows a rare event recorded on 24 May 2018 when the $N_{\text {res }}$ enhancement corresponds better with electric field at SP (blue) rather than with electric field at LS. Such situation might indicate relatively distant source region of the TGE from LS (more than $1 \mathrm{~km}$ ) that is closer to SP than to LS or an unusual charge distribution. It is interesting that the two strongest events that were recorded on 10 June 2017 and on 19 October 2018 occurred during extreme negative PG at LS and only moderate negative PG at SP. This might indicate that the LS was inside thundercloud, close to main lower negative charge center with a possible minor positive charge center below. Such a configuration is highly effective for downward acceleration of electrons. The charge structures inside thunderclouds might be very complicated and dynamic and often cannot be described by a simple vertical dipole model (Stolzenburg 1998; Saunders 2008). Two point measurements are still insufficient for reliable locations of charge centers.

It should also be mentioned that IC discharges were detected by EUCLID if a TGE was terminated or interrupted by lightning. These discharges were often multiple strokes, sometimes also composed of CG strokes. The information about lightning that interrupted or terminated the analyzed TGEs are summarized in Table 2, which also shows the changes of electric field at LS $\left(\Delta \mathrm{PG}_{\mathrm{LS}}\right)$ at the time of these discharges, maxima of $N_{\text {res }}$ for the individual events and values of $N_{\text {res }}$ at the times of the TGEs interruption by discharges $\left(N_{\text {res_int }}\right)$. The significant TGE on 19 October 2018 was not terminated by any lightning. On the other hand, the extreme event on 10 June 2017 was terminated by an extended multiple stroke discharge that occurred around the LS. The change of electric field at $L S\left(\triangle \mathrm{PG}_{\mathrm{LS}}\right)$ could not be evaluated for this event because a short data gap after the lightning. (Based on measurements at SP presented in Fig. 10, the change was likely positive). It should be noted that the $\Delta \mathrm{PG}_{\mathrm{LS}}$ are usually associated with the change of polarity of the vertical component of electric field. Thus, the change of electric field can reach larger values than the values that were measured before or after the discharge.

Our observation of TGEs is consistent with RREA mechanism and associated Bremsstrahlung as recently discussed by Bartoli et al. (2018) and Chilingarian (2018). The dependence of TGEs on the sign of vertical component of electric field, found in our observations and previous study by Kudela et al. (2017), was also discussed by Lidvansky (2003), Khaerdinov et al. (2005) and Bartoli 


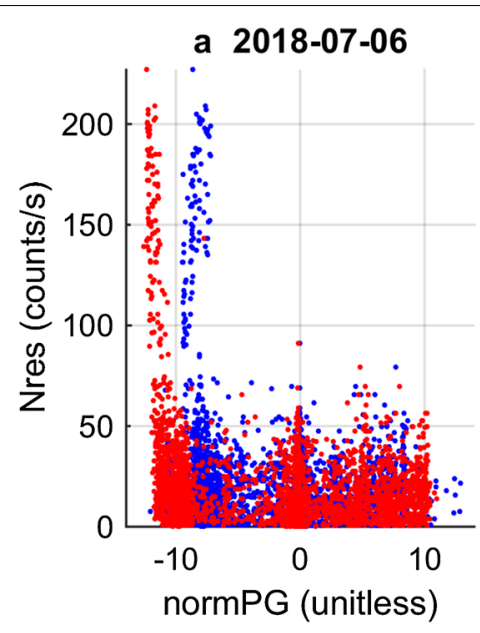

d 2018-10-19

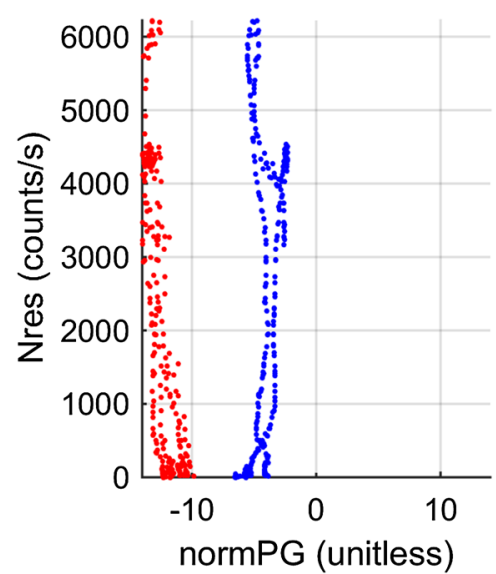

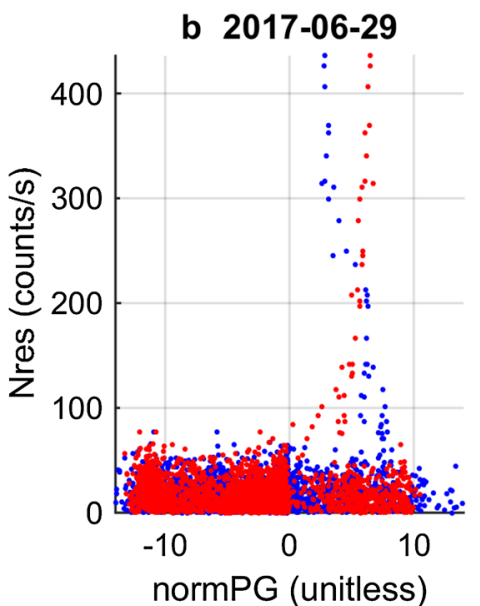

e 2017-05-30

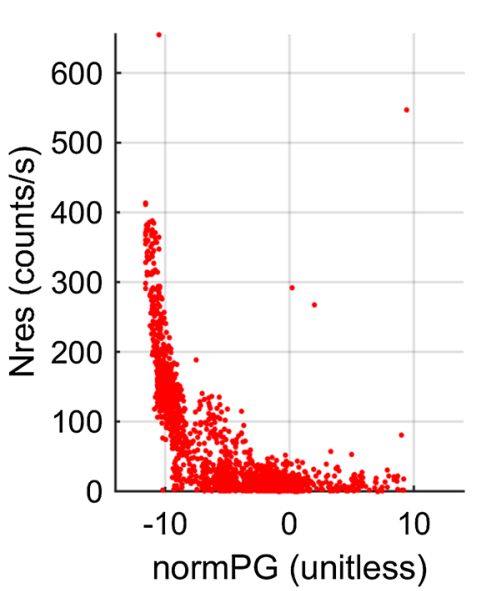

c 2018-05-24
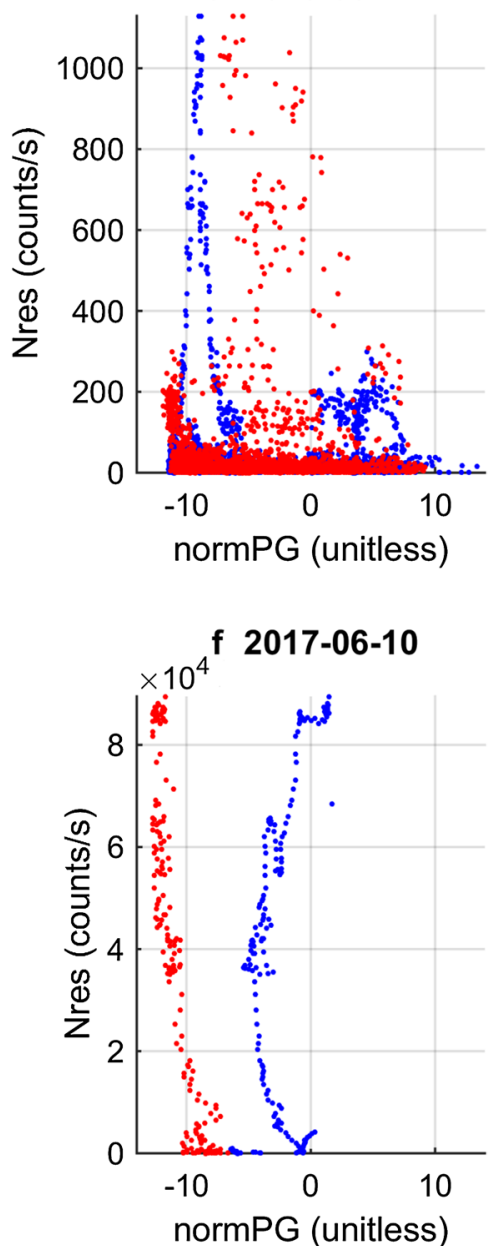

Fig. 12 Scatterplots between 1-s residual counts $N_{\text {res }}$ and normalized PG at Lomnicky peak (red) and Skalnate Pleso (blue) for the intervals (events) presented in Figs. 3, 4, 5, 6, 7 and 9

et al. (2018). Bartoli et al. (2018) analyzed observations in Tibet, China, at the altitude of $4300 \mathrm{~m}$ and performed simulations using CORSIKA code to investigate the development of secondary electrons and positrons following the hypothesis introduced by Zhou et al. (2016). They conclude that the enhancements of secondary cosmic rays are more frequent for negative PG because of the initial abundance of electrons with respect to positrons in the Earth's atmosphere. In addition, the primary positrons might have larger energies and be less influenced by the electric field. Bartoli et al. (2018) showed that there might be a minimum of counts for a specific positive PG and energy channel of the detector. Our measurements do not show reliably such a minimum. A minimum of occurrence rate of significant TGEs is visible in the histogram presented in Fig. 3c; however, the uncertainty (width) of its location on the PG axis is large. It should also be recalled that our observations at LS are at different latitude and altitude and that LS was often inside the thundercloud during the $N_{\text {res }}$ enhancements, whereas Bartoli et al. (2018) reported that the bottom of thunderclouds was typically $300 \mathrm{~m}$ above the ground (detector). Differences between individual events suggest that thickness, size and height of thunderstorm clouds around LS are highly variable.

Because of the effective energy threshold of the SEVAN channel $1(\sim 7-8 \mathrm{MeV})$ and because of the fact that the $N_{\text {res }}$ events are often terminated by a nearby lightning, it is likely that potential contribution from rain washout of radon 222 daughter isotopes (Suszcynsky et al. 1996) is negligible for the presented observation of $N_{\text {res }}$ enhancements. For the same reason, gamma-rays from electronpositron annihilation with energies of $0.511 \mathrm{MeV}$, studied by Enoto et al. (2017), can also be excluded.

The most important point of this study is the extreme event recorded on 10 June 2017. The enhancement in 
Table 2 Basic characteristic of the significant TGEs and lightning that terminated them

\begin{tabular}{|c|c|c|c|c|c|c|c|}
\hline Event date & $N_{\text {res_max }}($ counts/s) & $N_{\text {res_int }}$ (counts/s) & Discharge time (UT) & $\Delta P G_{L S}(k V / m)$ & $I_{\text {peak }}(\mathrm{kA})$ & Type & $\begin{array}{l}\text { Distance } \\
\text { from LS } \\
(\mathrm{km})\end{array}$ \\
\hline \multirow[t]{3}{*}{ 2018-07-06 } & 227 & 209 & $10: 03: 21.7$ & +261 & 9.7 & IC & 6.9 \\
\hline & & & $10: 03: 21.7$ & & 15.5 & IC & 6.7 \\
\hline & & & 10:03:21.7 & & 11.7 & IC & 6.3 \\
\hline 2017-06-29 & 436 & 436 & 15:54:04.9 & -174 & 11.0 & IC & 0.1 \\
\hline \multirow[t]{4}{*}{$2018-05-24$} & 1130 & 134 & 13:00:33.8 & +41 & -5.8 & IC & 2.6 \\
\hline & & & 13:00:33.8 & & -9.4 & IC & 2.9 \\
\hline & & & 13:00:34.0 & & -7.5 & IC & 2.3 \\
\hline & & & $13: 00: 34.2$ & & 38.3 & $C G$ & 2.8 \\
\hline 2018-10-19 & 6222 & None & None & None & None & None & None \\
\hline \multirow[t]{3}{*}{$2017-05-30$} & 413 & 292 & $14: 17: 17.5$ & +163 & 7.9 & IC & 3.5 \\
\hline & & & $14: 17: 17.6$ & & 7.9 & IC & 3.2 \\
\hline & & 655 & 14:27:08.6 & +225 & 6.8 & IC & 0.5 \\
\hline \multirow[t]{7}{*}{ 2017-06-10 } & 91,307 & 91,307 & $13: 14: 35.5$ & $+?$ & -18.5 & IC & 1.4 \\
\hline & & & $13: 14: 35.5$ & & -16.7 & $C G$ & 0.2 \\
\hline & & & 13:14:35.5 & & -21.5 & IC & 0.5 \\
\hline & & & $13: 14: 35.5$ & & 29.3 & $C G$ & 0.8 \\
\hline & & & 13:14:35.6 & & -6.8 & IC & 9.6 \\
\hline & & & 13:14:35.6 & & -6.2 & IC & 9.4 \\
\hline & & & $13: 14: 35.9$ & & -10.7 & IC & 0.8 \\
\hline
\end{tabular}

SEVAN channel 1 exceeded the background value more than 200 times and is to the best of authors' knowledge the strongest event recorded by SEVAN as only events that exceeded the background level several times were reported so far (Chilingarian 2014). It should be noted that the counts/s in SEVAN channel 1 reached almost 90,000 per second. Considering a typical pulse width of 7-8 $\mu$ s (measured by oscilloscope) and random occurrence of individual particles (pulses), the counts in channel 1 could be partly saturated (maximum counts, provided regular occurrence of pulses is around 130,000). The event was also observed by NM (Fig. 11). It should be noted that the neutron monitor is also partially sensitive to gamma-rays. The sensitivity of NM to gamma-rays increases with gamma-ray energy (Tsuchiya et al. 2012). However, the enhancements in the SEVAN combination $[0,1,0]$ also indicating neutrons and enhancement in the SEVAN $[1,0,1]$ coincidence indicating presence of highly energetic charged particles with energies that could reach $\sim 200 \mathrm{MeV}$ show that photonuclear reactions likely took place. A possible explanation for the neutron detection might be the photonuclear reactions ${ }^{14} \mathrm{~N}+\gamma \rightarrow{ }^{13} \mathrm{~N}+n$ or ${ }^{16} \mathrm{O}+\gamma \rightarrow{ }^{15} \mathrm{O}+n$ (Enoto et al. 2017), where $\gamma$ represents a gamma photon with energy exceeding the threshold of photonuclear reaction and $n$ neutron with initial energy given by the difference between photon energy and the threshold. The reaction with ${ }^{14} \mathrm{~N}$ is more probable because of higher concentration of nitrogen and lower energy threshold of the reaction with nitrogen. The thresholds are $10.55 \mathrm{MeV}$ for ${ }^{14} \mathrm{~N}$ and $15.7 \mathrm{MeV}$ for ${ }^{16} \mathrm{O}$. We cannot exclude that electron-induced reactions, mainly electro-disintegration ${ }^{14} \mathrm{~N}+e+E_{e} \rightarrow{ }^{13} \mathrm{~N}+n+e$, where $e$ denotes an electron and $E_{e}$ its kinetic energy with the threshold $10.55 \mathrm{MeV}$, could make a small contribution to the neutron production in the atmosphere (Babich et al. 2014). It should be noted that photonuclear can also take place in the material that surrounds the detector, e.g., in the roof, the threshold energy for ${ }^{56} \mathrm{Fe}$ to release neutrons is $11.2 \mathrm{MeV}$ (Tsuchiya 2014).

The neutron detections by NM64 and SEVAN, associated with TGE, were also observed at mount Aragats, Armenia (Chilingarian et al. 2010, 2012a). Compared with Aragats' detections, the extreme event recorded on 10 June 2017 at LS was much larger. Chilingarian et al. (2012a) reported maximum relative enhancement of $5.8 \%$ in NM and about $73 \%$ in SEVAN $[0,1,0]$ combination, whereas the relative enhancement detected by NM at LS was about $130 \%$ and more than $250 \%$ in the SEVAN $[0,1,0]$ combination.

Investigation of strong TGEs accompanied by photonuclear reactions might also find practical applications as the majority of neutrons produced in atmosphere disappear via neutron capture reaction ${ }^{14} \mathrm{~N}+n \rightarrow{ }^{14} \mathrm{C}+p$, 
where $p$ denotes a proton. The resulting isotope ${ }^{14} \mathrm{C}$ is quasi-stable with a half-life of 5730 years and is frequently used in radiocarbon dating (Babich and RousselDupré 2007; Enoto et al. 2017; Babich 2017). Obviously, the knowledge of ${ }^{14} \mathrm{C}$ production rate in the atmosphere is important for precise dating.

\section{Conclusions}

Enhancements of secondary cosmic ray flux (TGEs) were observed at the top of Lomnický Štít mountain (2634 m, Slovakia) in SEVAN channel 1 . The TGEs were usually (not only) recorded at times when large upward electric fields were measured at the same location. The electric field was also simultaneously measured at the slope of Lomnický štít at distance of about $1.86 \mathrm{~km}$. The electric fields at these locations often differed from event to event, which indicates that the TGEs were observed for different distances (possible up to more than $1 \mathrm{~km}$ ), sizes and positions of charge structures from Lomnický Stít. It was hypothesized that the two strongest TGEs occurred when the top of Lomnický Štít was close to the main negative charge center, possibly between the main negative charge center and minor lower positive charge region. The likely mechanism for the TGEs is acceleration of runaway electrons in high thunderstorm electric fields and subsequent Bremsstrahlung owing to their collisions with the air molecules. If a nearby lightning occurred, it abruptly terminated the event.

The observed enhancements lasted approximately from 1 to $15 \mathrm{~min}$ and usually exceeded the background values by several tens of percent. Enhancements that exceeded the background values several times were, however, also observed. The signals recorded in different SEVAN channels are consistent with arrival of particle (photon) flux from above, except for one strange event. The most interesting and strongest event exceeded the background values about 215 times and was also registered in other SEVAN channels and by the collocated neutron monitor that exhibited about $130 \%$ enhancement. The analysis shows that particles of energies sufficient for photonuclear reactions were generated during this TGE. A possible explanation for the likely detection of neutrons is an atmospheric photonuclear reaction, mainly ${ }^{14} \mathrm{~N}+\gamma \rightarrow{ }^{13} \mathrm{~N}+n$. A generation of neutrons in the roof above the neutron monitor and/or contribution of highenergy gamma-rays to the signal in neutron monitor cannot be excluded.

\footnotetext{
Abbreviations

CG: Cloud to ground; EFM: Electric field mill; IC: Intra-cloud; LS: Lomnický Štít; NM: Neutron monitor; PG: Potential gradient; SEVAN: Space environment viewing and analysis network; SP: Skalnaté Pleso; RREA: Relativistic runaway electron avalanches; TGE: Thunderstorm ground enhancements; TGF:Terrestrial gamma-ray flash.
}

\section{Acknowledgements}

Prof. Karel Kudela (in memoriam) is acknowledged for pioneering the research of cosmic rays at Lomnický Štít, for initiating this work and valuable discussion at the beginning of this study. Dušan Božík and Samuel Štefánik are acknowledged for the help with maintenance of measurements at SP and LS.

\section{Authors' contributions}

JC analyzed the data and wrote most of the paper. RL, MK and IS are responsible for the correct measurement by SEVAN and neutron monitor, data archiving and primary processing. JB is responsible for measurement of electric field and data archiving, GD is responsible for lightning data and their interpretation. JR helped with data interpretation and analysis. All authors read and approved the final manuscript.

\section{Funding}

The supports under the Grant 18-01969S by the Czech Science Foundation, under project SAV-18-04 between the Czech and Slovak Academy of Sciences, and under the Grant VEGA 2/0155/18 by the Slovak Academy of Sciences are acknowledged. R. Langer also acknowledges support under the project CRREAT, CZ.02.1.01/0.0/0.0/15_003/0000481, call number 02_15_003 of the Operational Programme Research, Development, and Education.

\section{Availability of data and materials}

Data from Skalnaté Pleso are available at http://datacenter.ufa.cas.cz/mlynky/ archive/Mlynek.SP/.

\section{Ethics approval and consent to participate}

Not applicable.

\section{Consent for publication}

Not applicable.

\section{Competing interests}

The authors declare that they have no competing interests.

\section{Author details}

${ }^{1}$ Institute of Atmospheric Physics of the Czech Academy of Sciences, Prague, Czech Republic. ${ }^{2}$ Institute of Experimental Physics, Slovak Academy of Sciences, Kosice, Slovakia. ${ }^{3}$ OVE Service GmbH, Dept., Vienna, Austria. ${ }^{4}$ Nuclear Physics Institute of the Czech Academy of Sciences, Husinec-Rez, Czech Republic.

Received: 11 October 2019 Accepted: 24 February 2020

Published online: 02 March 2020

\section{References}

Aiemsa-ad N, Ruffolo D, Saiz A, Mangeard P-S, Nutaro T, Nuntiyakul W et al (2015) Measurement and simulation of neutron monitor count rate dependence on surrounding structure. J Geophys Res Space Phys 120:5253-5265. https://doi.org/10.1002/2015JA021249

Babich LP (2006) Generation of neutrons in giant upward atmospheric discharges. JETP Lett 84:285-288. https://doi.org/10.1134/S002136400 6180020

Babich LP (2014) Fundamental processes capable of accounting for the neutron flux enhancements in thunderstorm atmosphere. J Exp Theor Phys 118(3):375-383. https://doi.org/10.1134/S1063776114030017

Babich LP (2017) Radiocarbon production by thunderstorms. Geophys Res Lett 44:11191-11200. https://doi.org/10.1002/2017gl075131

Babich LP, Roussel-Dupré RA (2007) Origin of neutron flux increases observed in correlation with lightning. J Geophys Res 112:D13303. https://doi. org/10.1029/2006JD008340

Babich LP, Bochkov E, Dwyer JR, Kutsyk IM, Zalyalov AN (2013a) Numerical analysis of 2010 high-mountain (Tien-Shan) experiment on observations of thunderstorm-related low-energy neutron emissions. J Geophys Res Space Phys 118:7905-7912. https://doi.org/10.1002/2013JA019261

Babich LP, Bochkov El, Kutsyk IM, Zalyalov AN (2013b) On amplifications of photonuclear neutron flux in thunderstorm atmosphere and possibility of detecting them. JETP Lett 97(6):291-296. https://doi.org/10.1134/S0021 364013060027 
Babich LP, Bochkov El, Kutsyk IM, Rassoul HK (2014) Analysis of fundamental interactions capable of producing neutrons in thunderstorm atmosphere. Phys Rev D 89:093010. https://doi.org/10.1103/PhysRevD.89.093010

Bartoli B et al (2018) Observation of the thunderstorm-related ground cosmic ray flux variations by ARGO-YBJ. Phys Rev D 97:042001

Bowers GS, Smith DM, Martinez-McKinney GF, Kamogawa M, Cummer SA, Dwyer JR et al (2017) Gamma ray signatures of neutrons from a terrestrial gamma ray flash. Geophys Res Lett 44:10063-10070. https://doi.org/10.1002/2017G L075071

Chilingarian A (2014) Thunderstorm ground enhancements-model and relation to lightning flashes. J Atmos Solar Terr Phys 107:68-76

Chilingarian A (2018) Long lasting low energy thunderstorm ground enhancements and possible Rn-222 daughter isotopes contamination. Phys Rev D 98:022007

Chilingarian A, Mkrtchyan H (2012) Role of the Lower Positive Charge Region (LPCR) in initiation of the Thunderstorm Ground Enhancements (TGEs). Phys Rev D 86:072003

Chilingarian A, Reymers A (2008) Investigations of the response of hybrid particle detectors for the Space Environmental Viewing and Analysis Network (SEVAN). Ann Geophys 26:249-257

Chilingarian A, Daryan A, Arakelyan K, Hovhannisyan A, Mailyan B, Melkumyan L, Hovsepyan G (2010) Ground-based observations of thunderstormcorrelated fluxes of high-energy electrons, gamma rays, and neutrons. Phys Rev D. 82:043009

Chilingarian A, Hovsepyan G, Hovhannisyan A (2011) Particle bursts from thunderclouds: natural particle accelerators above our heads. Phys Rev D 83:062001

Chilingarian A, Bostanjyan N, Vanyan L (2012a) Neutron bursts associated with thunderstorms. Phys Rev D 85:085017. https://doi.org/10.1103/PhysRevD85 085017

Chilingarian A, Bostanjyan N, Karapetyan T, Vanyan L (2012b) Remarks on recent results on neutron production during thunderstorms. Phys Rev D 86:093017

Chilingarian A, Chilingarian S, Hovsepyan G (2015) Calibration of particle detectors for secondary cosmic rays using gamma-ray beams from thunderclouds. Astropart Phys 69:37-43

Chilingarian A, Babayan V, Karapetyan T, Mailyan B, Sargsyan B, Zazyan M (2018) The SEVAN Worldwide network of particle detectors: 10 years of operation. Adv Space Res 61:2680-2696. https://doi.org/10.1016/j.asr.2018.02.030

Chum J, Diendorfer G, Šindelářová T, Baše J, Hruška F (2013) Infrasound pulses from lightning and electrostatic field changes: observation and discussion. J Geophys Res Atmos 118:10653-10664. https://doi.org/10.1002/jgrd.50805

Cummer SA, Briggs MS, Dwyer JR, Xiong S, Connaughton V, Fishman GJ et al (2014) The source altitude, electric current, and intrinsic brightness of terrestrial gamma ray flashes. Geophys Res Lett 41:8586-8593. https://doi. org/10.1002/2014GL062196

Diendorfer G, Pichler H, SchulzW (2015) LLS detection of upward initiated lightning flashes. In: 2015 Asia-Pacific international conference on lightning (APL), Nagoya, Japan

Dwyer JR, Uman MA (2014) The physics of lightning. Phys Rep 534(4):147-241

Eack KB, Beasley WH, Rust WD, Marshall TC, Stolzenburg M (1996) Initial results from simultaneous observation of $X$-rays and electric fields in a thunderstorm. J Geophys Res 101(D23):29637-29640. https://doi. org/10.1029/96JD01705

Enoto T et al (2017) Photonuclear reactions triggered by lightning discharge. Nature 551:481-484

Fishman GJ, Bhat PN, Mallozzi R, Horack JM, Koshut T, Kouveliotou C et al (1994) Discovery of intense gamma-ray flashes of atmospheric origin. Science 264:1313-1316

Gurevich AV, Milikh GM, Roussel-Dupre RA (1992) Runaway electron mechanism of air breakdown and preconditioning during a thunderstorm. Phys Lett $A$ 165(5-6):463-468. https://doi.org/10.1016/0375-9601(92)90348-P

Gurevich AV, Antonova VP, Chubenko AP, Karashtin AN, Mitko GG, Ptitsyn MO et al (2012) Strong flux of low-energy neutrons by thunderstorms. Phys Rev Lett 108(12):125001. https://doi.org/10.1103/PhysRevLett.108

Haldoupis C, Rycroft M, Williams E, Price C (2017) Is the "Earth-ionosphere capacitor" a valid component in the atmospheric global electric circuit?. J Atmos Solar Terr Phys 164:127-131
Hariharan B, Chandra A, Dugad SR et al (2019) Measurement of the electrical properties of a thundercloud through muon imaging by the GRAPES-3 experiment. Phys Rev Lett 122:105101

Ishtiaq PM, Mufti S, Darzi MA, Mir TA, Shah GN (2016) Observation of 2.45 MeV neutrons correlated with natural atmospheric lightning discharges by leadfree Gulmarg neutron monitor. J Geophys Res Atmos 121:692-703. https:// doi.org/10.1002/2015jd023343

Khaerdinov NS, Lidvansky AS, Petkov VB (2005) Electric field of thunderclouds and cosmic rays: evidence for acceleration of particles (runaway electrons). Atmos Res 1-4:346

Kudela K, Langer R (2009) Cosmic ray measurements in high Tatra mountains: 1957-2007. Adv Space Res 44(10):1166-1172

Kudela K, Chum J, Kollárik M, Langer R, Strhárský I, Baše J (2017) Correlations between secondary cosmic ray rates and strong electric fields at Lomnický štít. J Geophys Res Atmos. https://doi.org/10.1002/2016JD026439

Kuroda Y, Oguri S, Kato Y, Nakata R, Inoue Y, Ito C, Minowa M (2016) Observation of gamma ray bursts at ground level under the thunderclouds. Phys Lett $B$ 758:286-291

Lidvansky AS (2003) The effect of the electric field of the atmosphere on cosmic rays. J Phys G Nucl Part Phys 29:925-937

Martin IM, Alves MA (2010) Observation of a possible neutron burst associated with a lightning discharge. J Geophys Res 115:A00E11. https://doi. org/10.1029/2009ja014498

McCarthy M, Parks GK (1985) Further observations of X-rays inside thunderstorms. Geophys Res Lett 12:393-396. https://doi.org/10.1029/GL012i006p00393

Rycroft MJ, Israelsson S, Price C (2000) The global atmospheric electric circuit, solar activity and climate change. J Atmos Solar Terr Phys 62:1563-1576

Saunders C (2008) Charge separation mechanisms in clouds. Space Sci Rev 137:335-353. https://doi.org/10.1007/s11214-008-9345-0

Shah GN, Razdan H, Bhat GL, Ali GM (1985) Neutron generation in lightning bolts. Nature 313:773-775

Starodubtsev SA, Kozlov VI, Toropov AA, Mullayarov VA, Grigor'ev VG, Moiseev AV (2012) First experimental observations of neutron splashes under thunderclouds near the sea level. J Exp Theor Phys Lett 96:188-191

Stolzenburg M, Rust WD, Marshall TC (1998) Electrical structure in thunderstorm convective regions: 3. Synthesis. J Geophys Res 103:14097-14108

Suszcynsky DM, Roussel-Dupré R, Shaw G (1996) Ground-based search for X rays generated by thunderstorms and lightning. J Geophys Res 101(23):505

Torii T, Takeishi M, Hosono T (2002) Observation of gamma-ray dose increase associated with winter thunderstorm and lightning activity. J Geophys Res 107(D17):4324. https://doi.org/10.1029/2001JD000938

Torii T, Sugita T, Tanabe S, Kimura Y, Kamogawa M, Yajima K, Yasuda H (2009) Gradual increase of energetic radiation associated with thunderstorm activity at the top of Mt. Fuji. Geophys Res Lett 36:L13804

Torii T, Sugita T, Kamogawa M, Watanabe Y, Kusunoki K (2011) Migrating source of energetic radiation generated by thunderstorm activity. Geophys Res Lett 38:L24801. https://doi.org/10.1029/2011GL049731

Tsuchiya H (2014) Surrounding material effect on measurement of thunderstorm-related neutrons. Astropart Phys 57-58:33-38. https://doi. org/10.1016/j.astropartphys.2014.03.009

Tsuchiya $\mathrm{H}$ et al (2009) Observation of an energetic radiation burst from mountain-top thunderclouds. Phys Rev Lett 102:255003

Tsuchiya H, Enoto T, Yamada S, Yuasa T, Nakazawa K, Kitaguchi T et al (2011) Long duration Y ray emissions from 2007 and 2008 winter thunderstorms. J Geophys Res 116:D09113. https://doi.org/10.1029/2010JD015161

Tsuchiya H, Hibino K, Kawata K, Hotta N, Tateyama N, Ohnishi M et al (2012) Observation of thundercloud-related gamma rays and neutrons in Tibet. Phys Rev D 85:092006

Zhou XX, Wang XJ, Huang DH, Jia HY (2016) Effect of near-earth thunderstorms electric field on the intensity of ground cosmic ray positrons/electrons in Tibet. Astropart Phys 84:107-114. https://doi.org/10.1016/j.astropartp hys.2016.08.004

\section{Publisher's Note}

Springer Nature remains neutral with regard to jurisdictional claims in published maps and institutional affiliations. 